University of Louisville

ThinkIR: The University of Louisville's Institutional Repository

8-2014

\title{
Synthesis of a drug delivery vehicle for cancer treatment utilizing DNA-functionalized gold nanoparticles.
}

Tyler Brann

University of Louisville

Follow this and additional works at: https://ir.library.louisville.edu/etd

\section{Recommended Citation}

Brann, Tyler, "Synthesis of a drug delivery vehicle for cancer treatment utilizing DNA-functionalized gold nanoparticles." (2014). Electronic Theses and Dissertations. Paper 143.

https://doi.org/10.18297/etd/143

This Master's Thesis is brought to you for free and open access by ThinkIR: The University of Louisville's Institutional Repository. It has been accepted for inclusion in Electronic Theses and Dissertations by an authorized administrator of ThinkIR: The University of Louisville's Institutional Repository. This title appears here courtesy of the author, who has retained all other copyrights. For more information, please contact thinkir@louisville.edu. 
SYNTHESIS OF A DRUG DELIVERY VEHICLE FOR CANCER TREATMENT UTILIZING DNA-FUNCTIONALIZED GOLD NANOPARTICLES

\author{
By \\ Tyler Brann \\ B.S., University of Louisville, 2013

\begin{abstract}
A Thesis
Submitted to the Faculty of the

University of Louisville

J.B. Speed School of Engineering as Partial Fulfillment of the Requirements

for the Professional Degree
\end{abstract}

MASTER OF ENGINEERING

Department of Bioengineering

August 2014 


\section{SYNTHESIS OF A DRUG DELIVERY VEHICLE FOR CANCER TREATMENT UTILIZING DNA-FUNCTIONALIZED GOLD NANOPARTICLES}

Submitted by:

Tyler A. Brann

A Thesis Approved On

(Date)

By the Following Reading and Examination Committee

Dr. Martin G. O’Toole, Thesis Director

Dr. Robert S. Keynton

Dr. Xiao-An Fu 


\section{ACKNOWLEDGEMENTS}

The author would like to express his complete thanks and appreciation to everyone involved in the completion of this work and academic success as a whole. Thank you to Dr. Martin O'Toole for the opportunity to work on this project and for guidance through the whole process. Thank you to committee members, Dr. Robert Keynton and Dr. Xiaon-An Fu. Thank you to members of the O'Toole Lab; Kurtis James, Morgan Humphrey, Rajat Chauhan, Jon Sedelmeier, Betty Nunn, Samantha Manning, and Dhruvinkumar Patel. Thank you to Dr. Patricia Soucy and Dr. Archana Akalkotkar for their assistance with this work. Thank you to the Department of Bioengineering, to the J.B. Speed School of Engineering, and to the University of Louisville. Finally thank you to my friends and family for the never-ending support and encouragement. 


\begin{abstract}
The treatment of cancer with chemotherapeutic agents has made great strides in the last few decades but still introduces major systemic side effects. The potent drugs needed to kill cancer cells often cause irreparable damage to otherwise healthy organs leading to further morbidity and mortality. A therapy with intrinsic selective properties and/or an inducible activation has the potential to change the way cancer can be treated. Gold nanoparticles (GNPs) are biocompatible and chemically versatile tools that can be readily functionalized to serve as molecular vehicles. The ability of these particles to strongly absorb light with wavelengths in the therapeutic window combined with the heating effect of surface plasmon resonance makes them uniquely suited for noninvasive heating in biologic applications. Specially designed DNA aptamers have shown their ability to serve as drug carriers through intercalation as well as directly acting as therapeutic agents. By combining these separate molecules a multifaceted drug delivery vehicle can be created with great potential as a selective and controllable treatment for cancer.
\end{abstract}

Oligonucleotide-coated GNPs have been created using spherical GNPs but little work has been reported using gold nanoplates in this way. Using the Diasynth method gold nanoplates were produced to absorb strongly in the therapeutic near infrared (nIR) window. These particles were functionalized with two DNA oligonucleotides: one serving as an intercalation site for doxorubicin, and another, AS1411, serving directly as an anticancer targeting/therapeutic agent. These functional particles were fully synthesized and processed along with confirmation of DNA functionalization and doxorubicin intercalation. Doxorubicin is released via denaturation of the DNA structure into which doxorubicin is intercalated upon the heating of the gold nanoplate well above 
the DNA melting temperature. This temperature increase, due to light stimulation of surface plasmon resonance, was measured during laser application. Successful release of doxorubicin via laser application was measured with fluorescence measurements providing proof that the doxorubicin was successfully intercalated and released. 


\section{TABLE OF CONTENTS}

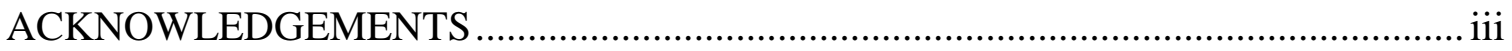

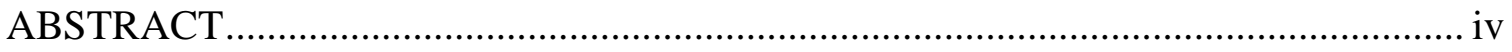

TABLE OF CONTENTS ....................................................................................... vi

NOMENCLATURE ……………………………......................................... vii

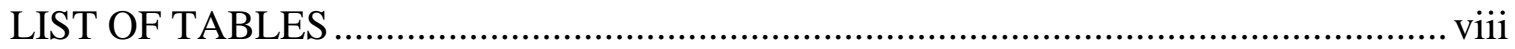

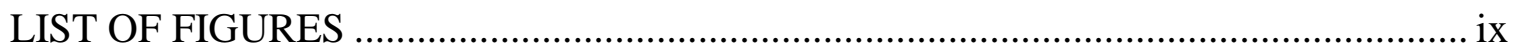

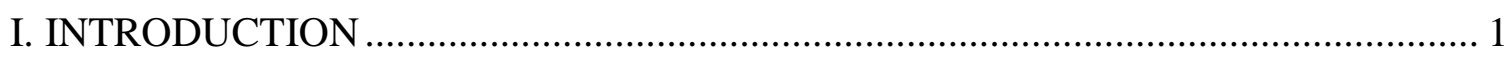

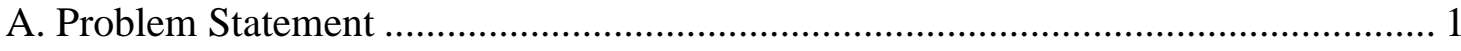

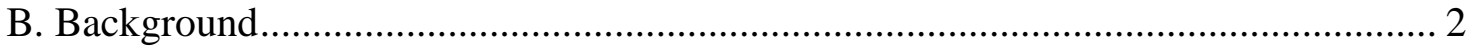

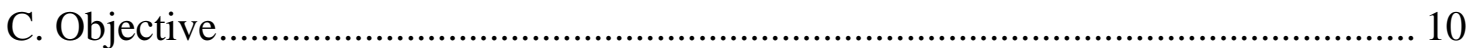

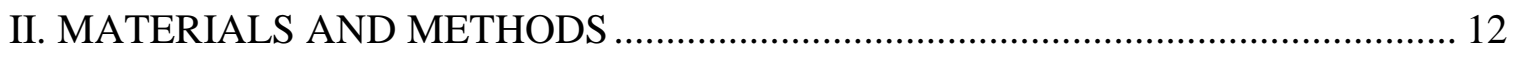

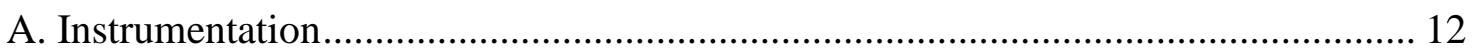

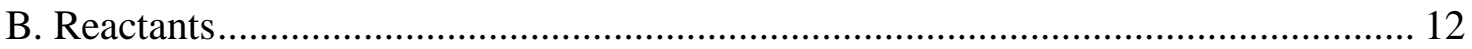

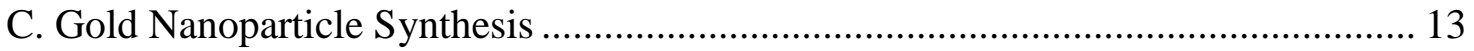

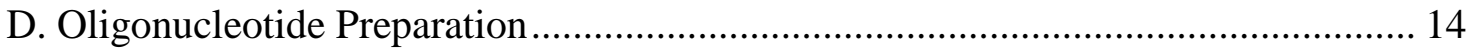

E. Functionalization of Gold Nanoplates with DNA …….......................................... 15

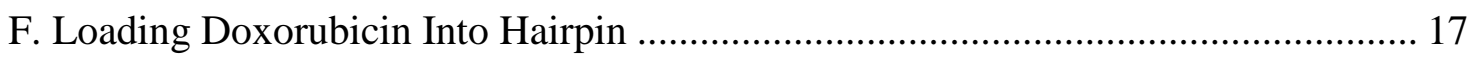

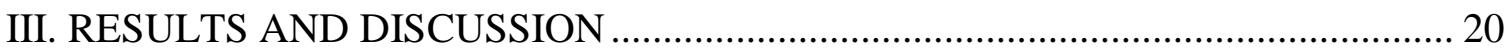

A. Gold Nanoparticle Synthesis ......................................................................... 20

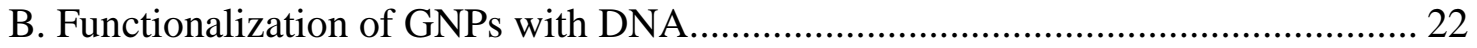

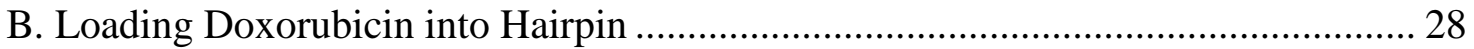

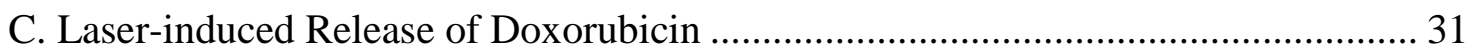

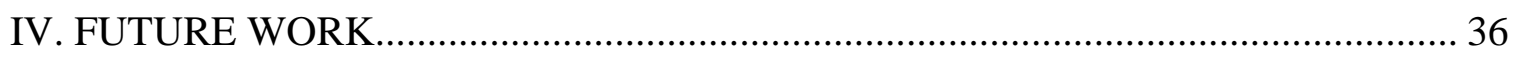

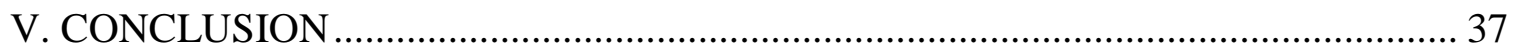

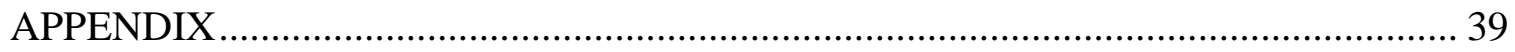

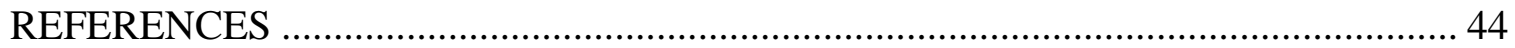




\section{NOMENCLATURE}

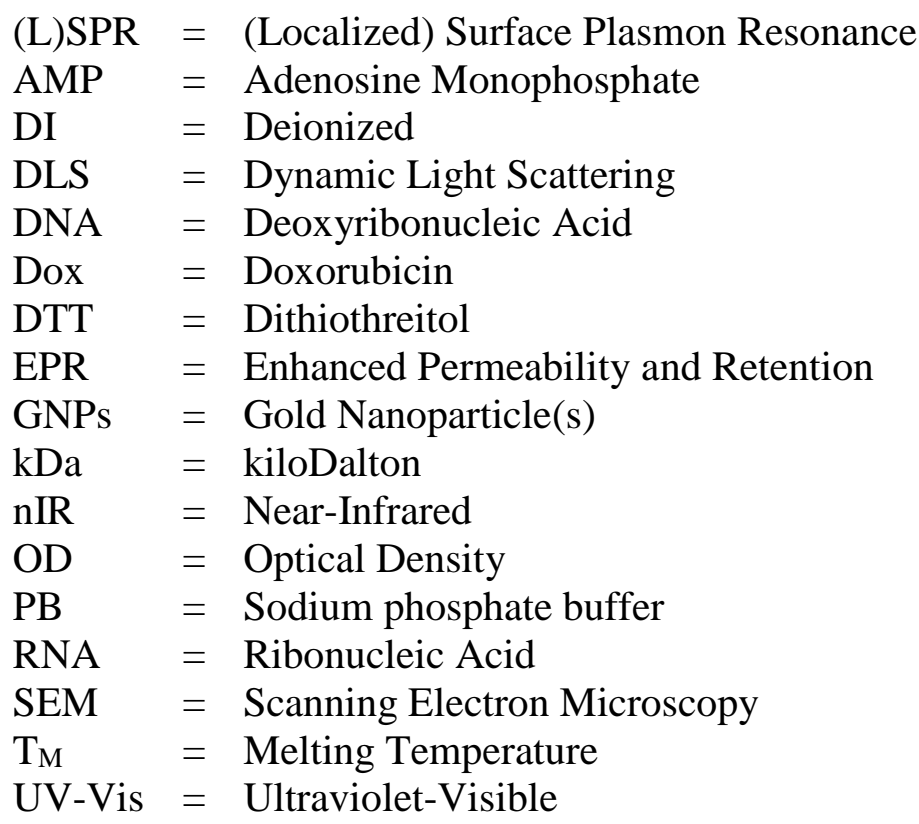




\section{LIST OF TABLES}

TABLE 1: OLIGONUCLEOTIDE SPECIFICATIONS 13

TABLE 2: SIZE AND ZETA POTENTIAL OF DNA-FUNCTIONALIZED GNPS ......23

TABLE 3: SIZE AND ZETA POTENTIAL OF AGGREGATED DNA-

FUNCTIONALIZED GNPS

TABLE 4: NUMBER OF HAIRPIN OLIGONUCLEOTIDES PER GOLD

NANOPLATE.

TABLE 5: MAXIMUM TEMPERATURE DURING LASER APPLICATION 


\section{LIST OF FIGURES}

FIGURE 1: The near-infrared window from 650-900 nm with minimal absorbance of biologic materials (Weissleder, 2001) .....................................................................

FIGURE 2: Quadruplex conformation of G-rich oligonucleotide allowing for aptameric interaction with cell proteins (Bates 2009) ...................................................................

FIGURE 3: Hairpin, or stem loop, structure. Intercalation of doxorubicin into occurs in $\mathrm{C}-\mathrm{G}$ rich regions

FIGURE 4: Chemical structure of Doxorubicin (Agudelo 2014) .......................................9

FIGURE 5: A. Conjugation of DNA oligonucleotides to gold nanoplate surface. B.

Loading of doxorubicin into the DNA hairpin. C. Laser induced release of doxorubicin.

FIGURE 6: UV-Vis spectrum of representative gold nanoparticles synthesized using the Diasynth method. The two peaks approximate the ratio of spheres and plates. Absorbance in therapeutic windows indicates suitability for laser absorption.

FIGURE 7: UV-Vis spectra of DNA-coated GNPs. The peaks shift slightly but maintain within the therapeutic window and absorbance at 817 is still suitable for laser absorption

FIGURE 8: UV-Vis spectra of aggregated DNA functionalized GNPs. No substantial NIR peak is visible indicating the high absorbance plates are no longer present. .27 FIGURE 9: UV-Vis spectra of GNP-Hairpin-AS1411 loaded with dox. Aggregation effects disproportionately affect the plates causing a decrease in plate to sphere absorbance ratio

FIGURE 10: Fluorescence readings of supernatants from lasered GNP-Hairpin samples loaded with dox. The first laser releases the most dox and subsequent lasering approaches the control. Laser light exposure was $817 \mathrm{~nm}$ wavelength at $800 \mathrm{~mW}$ for 5 minutes.........32 FIGURE 11: Fluorescence readings of supernatants from lasered GNP-Hairpin-AS1411 samples loaded with dox. The first laser releases the most dox and subsequent lasering approaches the control. Laser light exposure was $817 \mathrm{~nm}$ wavelength at $800 \mathrm{~mW}$ for 5

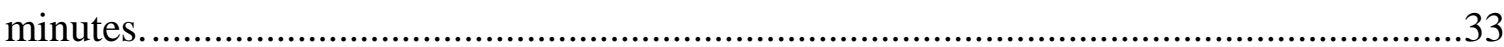

FIGURE 12: Residual plots for the size analysis of DNA loading...................................39

FIGURE 13: Residual plots for the zeta analysis of DNA loading. ..................................40

FIGURE 14: Residual plots for the size analysis of DNA loading...................................41

FIGURE 15: Residual plots for the size analysis of DNA loading...................................43

FIGURE 16: Fluorescence calibration curve for Doxorubicin..........................................43 


\section{INTRODUCTION}

\section{A. Problem Statement}

Gold nanoparticles (GNPs) have been studied for many years, due to their unique optical and physical characteristics, with the vast majority of research being done with gold nanospheres, the most common and easily produced particle type. More recently research has been done on more complex geometries of GNPs, such as nanoplates, which exhibit similar overall characteristics as gold nanospheres but with some outstanding features, such as high absorbance in the near-infrared (nIR) region of the electromagnetic spectrum. Due to high transmissivity of nIR light through biological media, this feature has proved invaluable for targeted photothermal ablation of living tissue with minimal collateral damage when targeting cancer cells (Gobin 2010). With the ability to reproducibly synthesize a large batch of gold nanoplates, using a technique such as Diasynth (Patel 2012), the unique therapeutic possibilities of these particles can be explored.

Conjugation of GNPs with various macromolecules has been studied for the ability to provide stability as well as drug delivery options to create versatile platforms for biological applications (Delong 2010). One such biomolecule that has become popular for this application in recent years is oligonucleotides. The application of DNA/RNA strands to create tertiary structures such as stem loops, or hairpins, has also been used independently to create biologically compatible drug delivery platforms through intercalation of therapeutic agents (Agudelo 2014). These drugs can then be released through thermal, chemical, or enzymatic denaturation of the DNA. Still other sequences of single stranded DNA, such as the guanosine-rich aptamer AS1411, have been found to 
have direct therapeutic effects against cancer cells (Stahel 2003, Bates 2009). The method of action of AS1411 is through geometric recognition with cell surface proteins only present on cancer cells, making AS1411 effectively inert to noncancerous cells. The clinical potential of AS1411 has been curtailed through short systemic circulation time.

By conjugating a hairpin structure to the surface of a gold nanoparticle, thermal denaturation can be achieved by heating the gold through application of external light to induce localized surface plasmon resonance (Gobin 2010). DNA-functionalization of GNPs, intercalation of dox into hairpin DNA, and creation of therapeutic DNA aptamers alone have been accomplished. Research has been broad with applications ranging from creating higher order supramolecular structures, selective binding of aptamers to cells or other DNA, or use as diagnostic marker for biosensing (Song 2010, Delong 2010). Each has shown some success as a tool for cancer therapy but not without limitations. A single complex combining all of these types of therapeutic modalities has the potential to bring a multifaceted approach to cancer therapy. This would include the selective molecular effects of the AS1411, the induced drug release of the hairpin, and the thermal effect of the GNPs.

\section{B. Background}

Cancer has been one of the largest causes of mortality globally and the number of cancer cases is continually rising (Bode 2009). Standard treatments for cancer consist of either radiation, chemotherapy, or both, which can have devastating side effects (Chabner 2005). One of the largest drawbacks of chemotherapy is the lack of specificity and controllability in the effect of the drug, often leading to dose-limited application that risks severe damage to otherwise healthy organs. It is desirable to have a treatment that could be deployed such that the active drug would remain sequestered until released via a controlled 
secondary mechanism, or a drug that exhibits specificity in only damaging the cancerous tissue.

GNPs have been used for decades in biological applications for the detection, diagnosis, and treatment of various diseases, utilizing the unique chemical and optical properties of nano-sized gold. They can also cover a broad spectrum of sizes, geometries (including spheres, rods, and plates), and compositions, each of which has unique characteristics. For biological applications GNPs are useful for their small size, biocompatibility, and ability to be conjugated easily with other molecules (Delong 2010). Sizes of GNPs can range from one nanometer to over 120 nanometers (Boisselier 2009), but for therapeutic applications size is most immediately relevant to the ability of the particles to reach the desired region in the body. In the case of intravenous cancer treatment the minimum size would be directly dependent on the perforations of the endothelium providing tumor perfusion (Iyer 2006), which have been shown to be larger in tumor vasculature than healthy vessels, a phenomenon referred to as the enhanced permeability and retention effect, or EPR.

The major determining factor for particle geometry is the chemical synthesis and resulting composition of the GNPs. Through the reaction of a gold salt, tetrachloroauric acid, with sodium thiosulfate in a cellulose acetate dialysis membrane non-spherical gold particles can be created in a one-step reaction without the use of seeds or other capping agents. Furthermore, using the "Diasynth" method gold nanoplates can be synthesized to be tuned to a desired size, directly corresponding to a maximum absorbance wavelength in the near-infrared range, by controlling the surface area of cellulose membrane and reaction temperature (James 2013, Patel 2012). Gold nanoplates are the main product of this 
reaction although smaller spherical GNPs are also formed, and can be removed through physical separation (Zhang 2012). Generally gold nanoplate dimensions measure from 40 $\mathrm{nm}$ to $1 \mathrm{um}$ edge length and 5 to $50 \mathrm{~nm}$ thick with the possibility of truncation of the vertices. Synthesis in solution usually produces single crystalline face-centered cubic lattices. Edge length, thickness, and truncation all ultimately affect the light extinction properties, and are to some degree controllable (Millstone 2009).

The most important property of GNPs for cancer therapy is a phenomenon known as localized surface plasmon resonance, or LSPR. Localized surface plasmon resonance is a well-studied ability of colloidal metal particles to vibrate at high frequency when exposed to an electromagnetic field. This is caused by the creation of an electric dipole within the particle, due to a difference in electron density, causing the particle to oscillate in turn with the frequency of the electric field of the incoming light. The application of this effect is even further magnified in potential when it is coupled with the near-infrared transparency of human skin. The wavelength range from 650 to $950 \mathrm{~nm}$ is known as the optical, or therapeutic, window for biomedical applications due to the dip in absorbance seen for many biologic media as seen in FIGURE 1. The bounds of this window consist of oxygenated and deoxygenated hemoglobin increasing in absorbance at the visible end of the window and water increasing at the infrared end. This indicates not only will minimal absorbance be seen in this window but maximum transmission as well. Utilizing the LSPR effect within the range of the therapeutic window allows for optimal excitation of GNPs with minimal damage to other tissue. Studies have shown the use of GNPs in this way for the treatment of tumors in mice (Gobin 2010 and Hirsch 2003). 


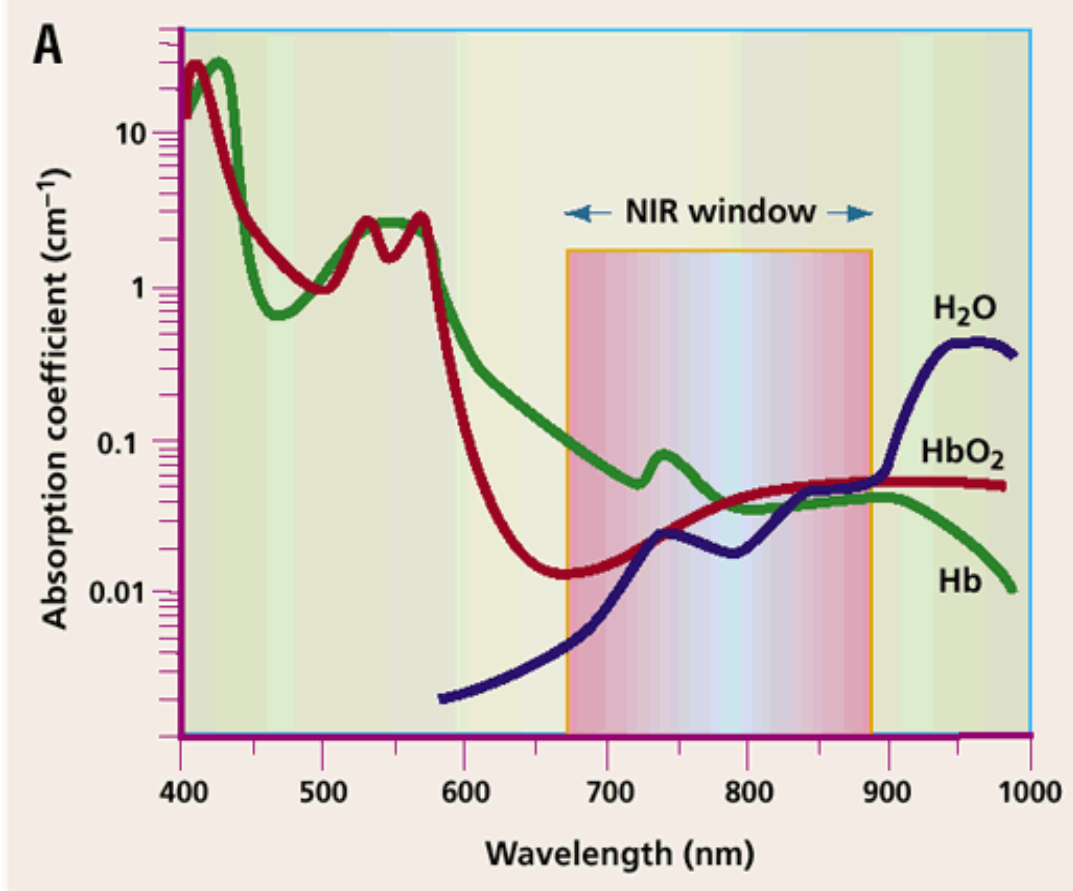

FIGURE 1 The near-infrared window from 650-900 nm with minimal absorbance of biologic materials (Weissleder, 2001)

To enhance the abilities of GNPs various studies have looked at the potential of forming functional layers on the exterior of the gold surface. Conjugation of GNPs with a variety of molecules has been researched. The high affinity binding of sulfhydryl, or thiol, groups for gold surfaces, has been heavily exploited to allow for coating with thiolated molecules such as polyethylene glycol, DNA, or RNA (Ghosh 2008). Through functionalization with these molecules the particles can become more resistant to aggregation, self-assemble to form larger structures such as lattices, or serve as vectors for gene or drug delivery (Witten 2008, Delong 2010). Characterization of DNAfunctionalized spherical GNPs has been reported rather extensively utilizing techniques including SEM imaging, optical spectroscopy, dynamic light scattering, and 
electrophoresis. Additionally, drug release from coated spherical GNPs has been studied both with and without in vitro cell studies analyzing cytotoxicity. However, most of this research has been done using spherical GNPs (Delong 2010). The use of gold nanoplates in this manner has much fewer reports but the approach in principle is identical.

DNA aptamers are functional structures composed of nucleotides or peptides to bind to specific molecules or enzymes. They are similar in action to antibodies, which can inhibit signal pathways or signal for immune response to cancer cells, using a key-and-lock type site of recognition. Aptamers, being composed of nucleotides or peptides, can still bind to surface receptors but can also disrupt transcription and translation processes. One molecule that has already shown great promise as a standalone cancer treatment is the DNA aptamer AS1411 (Bates 2009). The sequence, discovered and developed by Bates et al., consisting of a guanosine-rich oligonucleotide chain that has been shown to form quadruplex structures known as G-quartets, as seen in FIGURE 2, which can have protein binding properties. Physiologically these structures have shown several unique characteristics that give them great potential as a chemotherapeutic agent. While AS1411 exhibits decreased vulnerability to nuclease activity, increased uptake by cancer cells, and anti-proliferative effects the mechanisms of action for the molecule has been and continues to be studied. The current understanding of the mechanism concludes that nucleolin protein is displayed primarily on the surface of cancer cells, while only in the nucleus of healthy cells. AS1411 binds to nucleolin on the surface, which allows for internalization of the nucleolin-AS1411 complex. The AS1411 then causes further disruption to nucleolin dependent pathways linked to normal cancer progression. In vitro studies have shown AS1411 effective in treating a variety of cancer types across different cell lines. Initial 
clinical trials have been performed showing no adverse effects while proving effective in stabilization or even complete response. Further clinical testing is underway and the study of the mechanisms involved continue to advance.

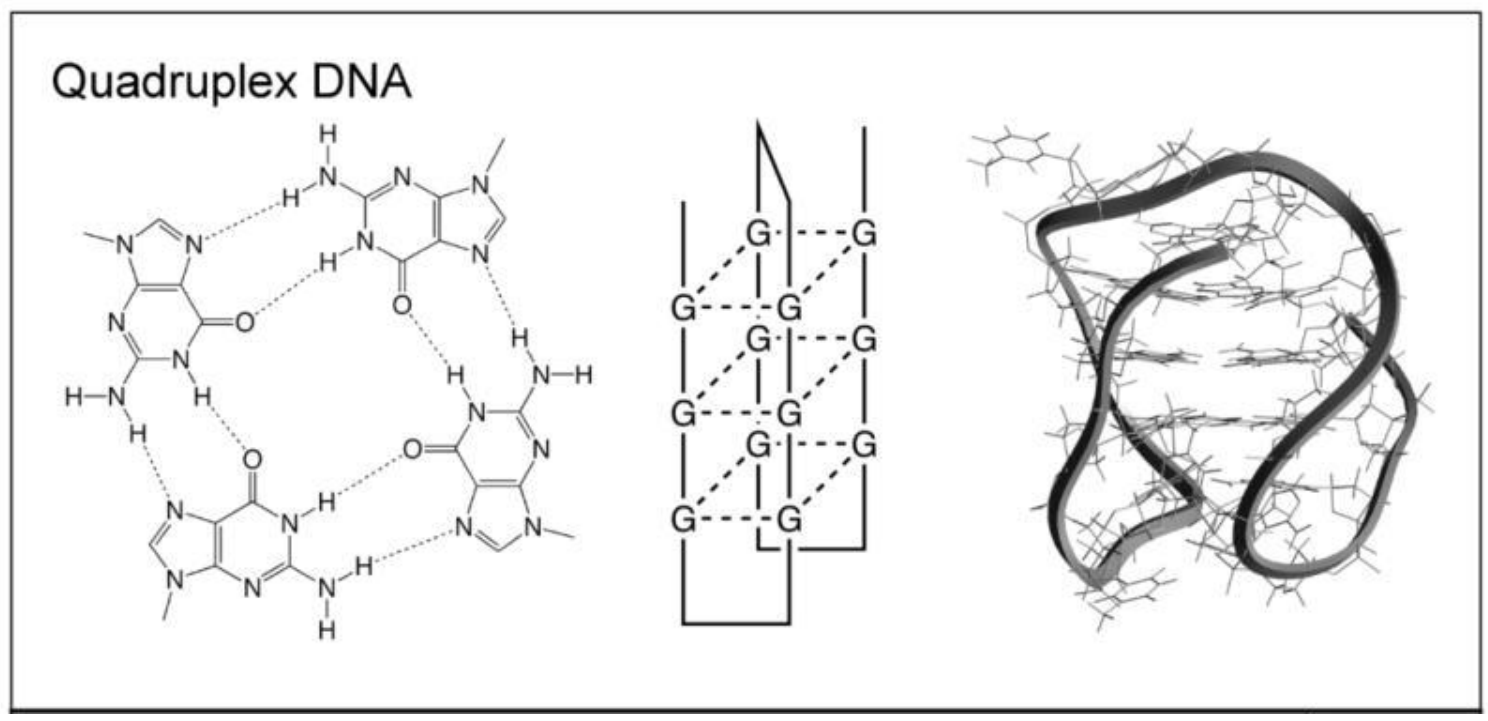

FIGURE 2 Quadruplex conformation of G-rich oligonucleotide allowing for aptameric interaction with cell proteins (Bates 2009)

Another functional DNA molecule that has been studied extensively is the stem loop, or hairpin. By controlling the sequence of nucleotides on a single DNA strand selfcomplementary segments can be arranged. When allowed to fold these segments bind to each other through traditional Watson-Crick base pairing, creating a double stranded structure from a single DNA strand as seen in FIGURE 3. Using this type of automatic folding can yield complex structures with multiple loops as well as simple short sequences with a single loop. Hairpins have been applied as molecular beacons, suppressors of polymerase chain reactions, high-sensitivity probes, and drug carriers (Walter 1998, Broude 2002). Chemotherapeutic agents that intercalate between the base pairs of double- 
stranded DNA can be loaded into the stem region of the hairpin. Extreme changes in the environment such as temperature, $\mathrm{pH}$, or salt concentration causes destabilization of the base pairing and denaturing of the hairpin structure. Using this effect the hairpin can be used as a selective release vehicle of active drugs.

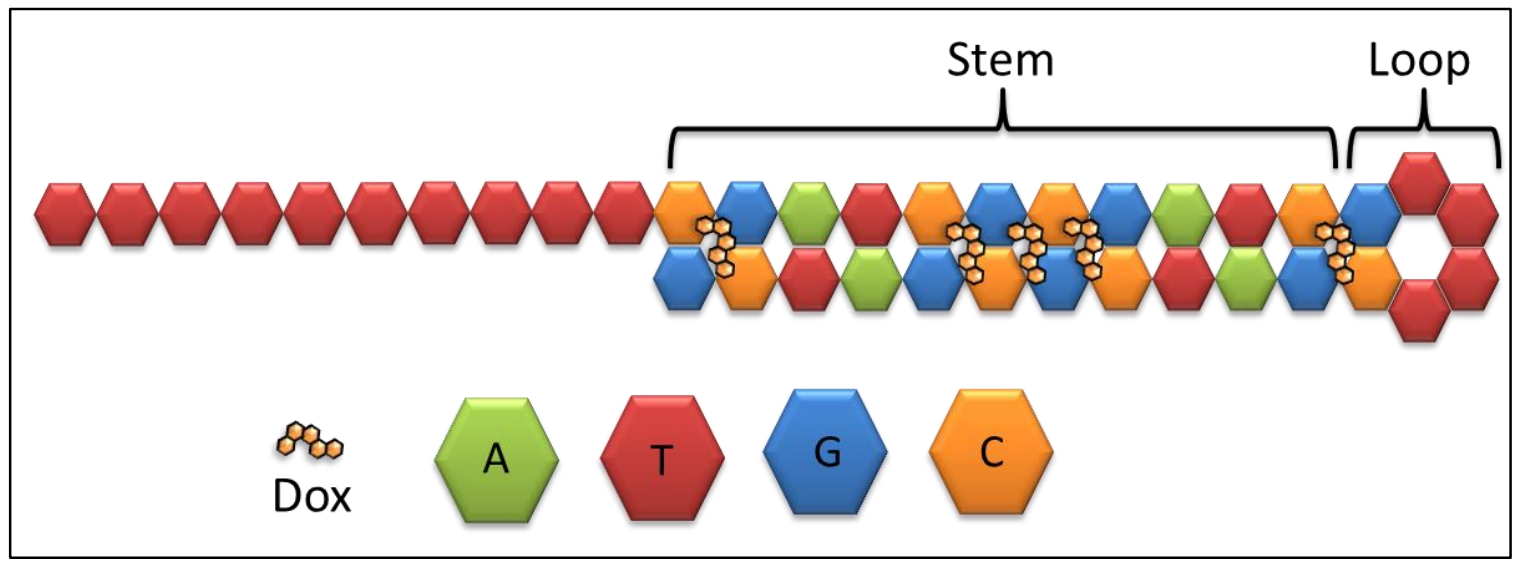

FIGURE 3 Hairpin, or stem loop, structure. Intercalation of doxorubicin into occurs in C$\mathrm{G}$ rich regions.

Doxorubicin is a common chemotherapy drug from a family known as anthracyclines, and FIGURE 4 shows its chemical structure. It can be used for many types of cancer and is often included in combination with other chemotherapeutic agents. Delivery of doxorubicin is typically intravenous and can be aided by pegylation and/or encapsulation in liposomes (Tacar 2013). Systemic exposure to doxorubicin can cause fatal toxicity to the heart as well as significant toxicity to the brain, liver and kidneys (Chatterjee 2010). Due to these side effects the dosage of doxorubicin must be limited and other additional therapies must be employed. A more selective release of doxorubicin would reduce these side effects while still allowing for cancer cell destruction. The method of action of doxorubicin is generally through intercalation with DNA and DNA-associated 
enzymes (Tacar 2013). This causes the halting of replication and transcription, effectively halting cell proliferation, while also producing free radicals that cause further damage to DNA and protein production. A secondary effect of these disturbances is the reduction in cellular energy production and initiation of autophagy. Multiple other chemical pathways, such as AMP-activated protein kinase and Bcl-2/Bax, are inhibited or activated by doxorubicin and are known to control programmed cell death, or apoptosis (Tacar 2013).

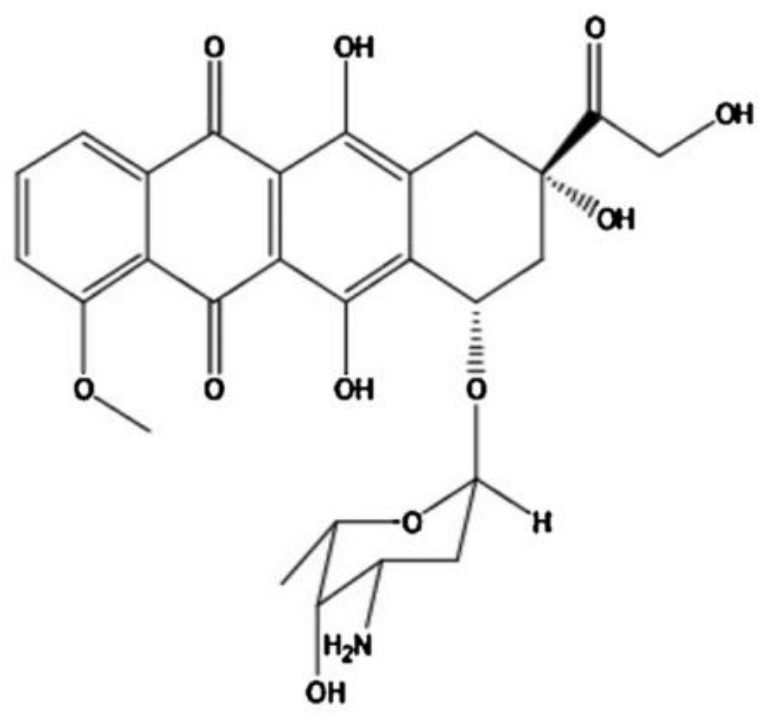

Doxorubicin

FIGURE 4 Chemical structure of Doxorubicin (Agudelo 2014)

The double helix structure of DNA, with major and minor grooves and stacked base pairs, allows for the binding of many different molecules. In the case of doxorubicin the binding occurs due to the ability of the aromatic rings to generate a pi-stacking arrangement between consecutive bases while the amine group remains within the minor groove. Optimal binding has been found to occur in regions rich in cytosine-guanosine base pairs as shown in FIGURE 3, specifically with repetition of the sequence CGATCG (Frederick, 
1990). Using a DNA hairpin in this manner acts as a biocompatible carrier for doxorubicin with the ability to denature with increases in temperature, thereby releasing the drug.

\section{Objective}

The objective of this work is to produce a versatile and selective vehicle for the delivery and release of chemotherapeutic agents to the site of cancer cells. The overall process is depicted in FIGURE 5. Utilizing the previously mentioned Diasynth method to obtain gold nanoplates, a drug delivery vehicle is created through the use of single stranded DNA molecules and an intercalating drug, doxorubicin. Release of doxorubicin is induced via nIR laser application to heat gold nanoplates and denature the hairpin DNA. The synthesis and processing of this vehicle is detailed as well as testing to quantify the doxorubicin loading capacity, the bulk solution temperature during laser application, and the release of doxorubicin as a function of successive of laser exposure. 


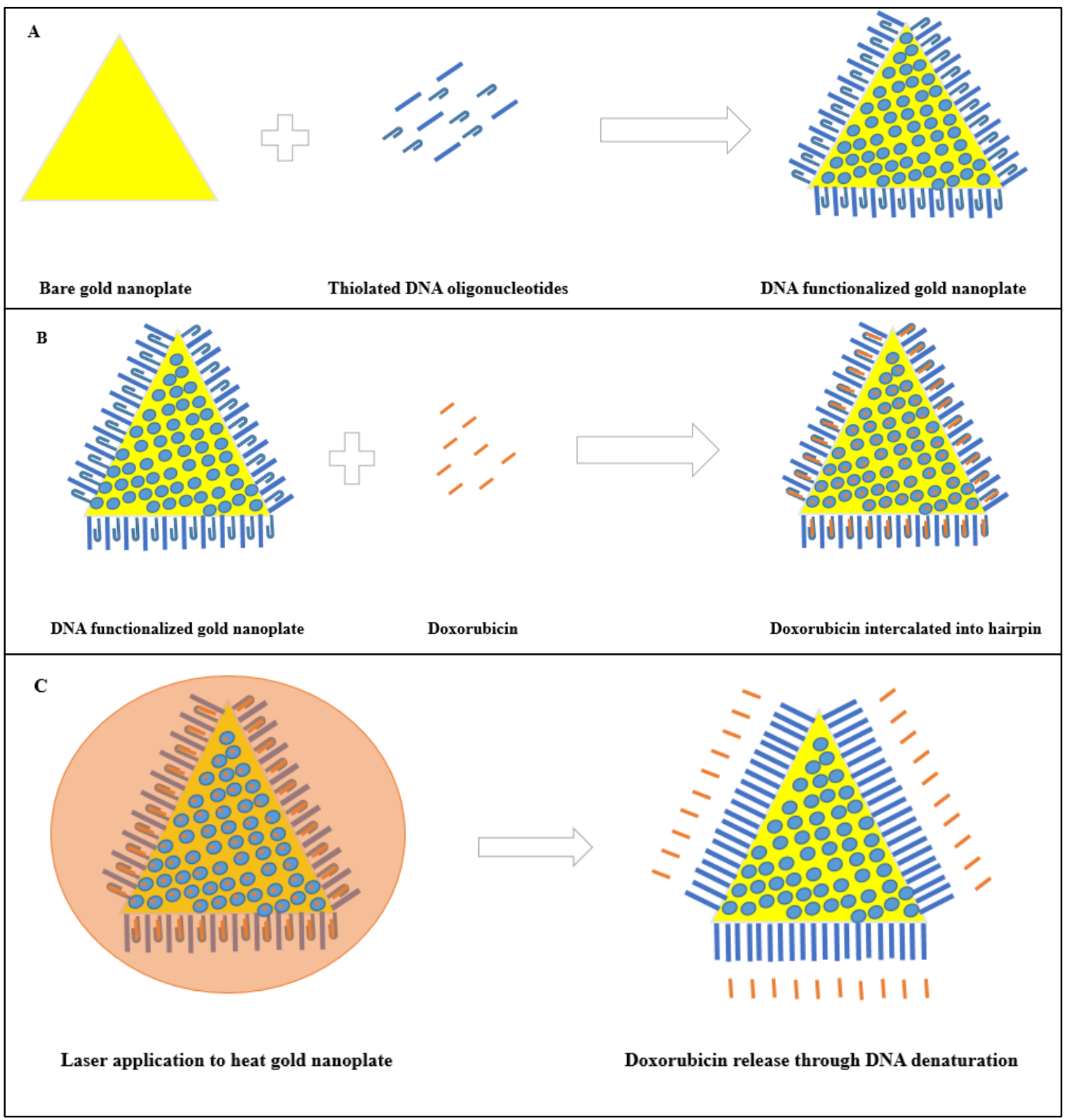

FIGURE 5 A. Conjugation of DNA oligonucleotides to gold nanoplate surface. B. Loading of doxorubicin into the DNA hairpin. C. Laser induced release of doxorubicin. 


\section{MATERIALS AND METHODS}

\section{A. Instrumentation}

All absorbance spectra were measured on a Varian (Palo Alto, CA) Cary 50 Bio UV-Vis Spectrophotometer using $12 \mathrm{~mm}$ quartz cuvettes and at a 10x dilution in Nanopure water, unless otherwise stated. Zeta potential and particle sizes (Dynamic Light Scattering, DLS) were measured using a Malvern Instruments (Worcestershire, United Kingdom) Zetasizer Nano ZS90, at a 10x dilution unless otherwise stated. Fluorescence was measured with a Turner Biosystems (Promega, Madison, WI) Modulus Fluorometer using the green fluorescence module accessory and glass microcell cuvettes with all readings being undiluted unless noted. The laser used for heating of the particles was a Coherent Inc (Santa Clara, CA) FAP System Model with a fixed emission wavelength of $817 \mathrm{~nm}$ and variable power setting. Actual laser power was measured before lasering using a Coherent FieldMax II-TO. The temperature of the solution while lasering was measured using an Omega Engineering (Stamford, CT) Thermocouple Data Logger (OM-EL-USB-TC-USB) and Omegasoft Data Logger Software (Rev. 6.8) using the provided type K thermocouple.

\section{B. Reactants}

The gold salt used was hydrogen tetrachloroaurate-III trihydrate $\left(\mathrm{HAuCl}_{3} * 3 \mathrm{H}_{2} \mathrm{O}\right)$ purchased from Alfa Aesar (Ward Hill, MA 01835). A $1.76 \mathrm{mM}$ solution was prepared by dissolving 1 gram in $14.725 \mathrm{~L}$ of DI water in a volumetric flask protected from light with aluminum foil. Sodium thiosulfate pentahydrate $\left(\mathrm{Na}_{2} \mathrm{~S}_{2} \mathrm{O}_{3} * 5 \mathrm{H}_{2} \mathrm{O}\right)$ was purchased from Sigma-Aldrich (St. Louis, MO) and $186.1 \mathrm{mg}$ was used to create a $250 \mathrm{~mL}$ solution at 3 mM. Oligonucleotides were purchased from Integrated DNA Technologies (Coralville, IA) and dissolved in Nanopure water to create stock solutions between 10 and $1 \mathrm{uM}$. See 
TABLE 1 for oligonucleotide specifications. Dithiothreitol $\left(\mathrm{C}_{4} \mathrm{H}_{10} \mathrm{O}_{2} \mathrm{~S}_{2}\right)$ at $1 \mathrm{M}$ concentration in $\mathrm{H}_{2} \mathrm{O}$ and dry powder doxorubicin hydrochloride $\left(\mathrm{C}_{27} \mathrm{H}_{29} \mathrm{NO}_{11} * \mathrm{HCl}\right)$ were purchased from Sigma-Aldrich (St. Louis, MI 63103).

TABLE 1: OLIGONUCLEOTIDE SPECIFICATIONS

\begin{tabular}{|c|c|c|c|c|}
\hline DNA & Modifiers & Conformation & $\begin{array}{l}\text { Melting } \\
\text { Temperature }\left({ }^{\circ} \mathrm{C}\right)\end{array}$ & $\begin{array}{l}\text { Extinction } \\
\text { Coefficient at } \\
260 \mathrm{~nm} \\
(\mathrm{~L} /(\mathrm{mol}-\mathrm{cm})) \\
\end{array}$ \\
\hline Hairpin & 3'Thiol & $\begin{array}{l}\text { Hairpin/Stem } \\
\text { Loop }\end{array}$ & 69.7 & 453,600 \\
\hline \multicolumn{5}{|c|}{$\begin{array}{l}\text { Sequence: } 5^{\prime} \text {-AGC TAG CGC TAG CGC TAG CTT TTG CTA CGC CTA GCG CTA } \\
\text { GCT TTT TTT TTT /3ThiolMC3-D/ -3' }\end{array}$} \\
\hline AS1411 & 5' Thiol & Quadruplex & 67.0 & 298,000 \\
\hline \multicolumn{5}{|c|}{$\begin{array}{l}\text { Sequence: 5'- /5ThiolMC6-D/TTT TTT GGT GGT GGT GGT TGT GGT GGT GGT } \\
\text { GG -3' }\end{array}$} \\
\hline
\end{tabular}

\section{Gold Nanoparticle Synthesis}

GNPs were synthesized using the Diasynth process (Patel 2012) and reaction parameters were chosen to yield particles with maximum absorbance at approximately 820 nm (James 2013) to match the wavelength at which the laser emits. Both temperature and surface area to volume ratio of the membrane can be varied to tune the maximum wavelength absorbance. The dialysis tubing cellulose membrane used was $43 \mathrm{~mm}$ wide 
and had a $12 \mathrm{kDa}$ molecular weight cutoff, purchased from Sigma-Aldrich and stored in DI water in a refrigerator at $4^{\circ} \mathrm{C}$. Before use each membrane is cut to size and rinsed thoroughly with DI water. A membrane of $12 \mathrm{~cm}$ is taken and clipped on one end with a plastic dialysis clip. Then $32.6 \mathrm{~mL}$ of $1.76 \mathrm{mM}$ gold salt solution is added followed by quickly adding $7.4 \mathrm{~mL}$ of $3 \mathrm{mM}$ sodium thiosulfate solution and agitating for 5 seconds. The membrane is then clipped on the other end while minimizing air bubbles within the membrane, giving a final effective membrane length of $10 \mathrm{~cm}$. The closed membrane is then submerged in a bath of DI water of at least $25 \mathrm{~L}$ circulated and maintained at $27^{\circ} \mathrm{C}$ by a Thermo (Waltham, MA) NESLAB RTE-221 Circulator. After reacting for 1 hour the membrane is removed and the solution is emptied into a $40 \mathrm{~mL}$ plastic tube. The UV-Vis spectrum of the product is analyzed to verify the peak absorbance wavelength, and size (DLS) and zeta potential are also measured.

\section{Oligonucleotide Preparation}

Lyophilized oligonucleotides were used to create stock solutions in Nanopure water. Using the provided molecular weights as provided by the supplier, $10425.9 \mathrm{~g} / \mathrm{mol}$ and $15839.4 \mathrm{~g} / \mathrm{mol}$ for AS1411 and Hairpin respectively, $7.7 \mathrm{uM}$ and $5 \mathrm{uM}$ solutions were created respectively. Each oligonucleotide solution is stored in $4{ }^{\circ} \mathrm{C}$ with the thiol modification containing a disulfide bond. To become activated the disulfide bond must be broken to form a sulfhydryl that will readily react with the gold surface. For this dithiothreitol, DTT, is used to cleave the disulfide bond, requiring each DNA solution react in $0.1 \mathrm{M}$ DTT concentration for at least 1 hour. Immediately prior to deprotection the DNA is exposed to a sodium phosphate buffer $(\mathrm{PB})$ environment, at $0.18 \mathrm{M}(\mathrm{pH} 8)$, and heated 
to $90^{\circ} \mathrm{C}$, well above the melting temperature of the DNA strands, and then allowed to cool. A salt concentration, provided by the buffer, is needed to provide the proper base pairing of DNA. Annealing in this way denatures the DNA and then allows it to fold in the presence of the buffer ensuring folding before bonding to the gold. To obtain the purified, activated oligonucleotides the excess DTT must be separated using a chromatography column. Using GE (Buckinghamshire, United Kingdom) illustra NAP-25 columns and $10 \mathrm{mM} \mathrm{PB} \mathrm{(pH} \mathrm{8)}$ as the mobile phase the two oligonucleotides are eluted. Each sample of oligonucleotide is then analyzed with UV-Vis spectroscopy to determine the absorbance at $260 \mathrm{~nm}$ which correlates to the concentration of DNA by using the manufacturer-supplied molar absorptivity for each strand of DNA.

\section{E. Functionalization of Gold Nanoplates with DNA}

Once the oligonucleotides are separated from the DTT via the chromatography columns, addition of gold particles must take place within a short period of time. Once the DTT is removed the activated sulfhydryl groups on the oligonucleotides can reform disulfide bonds with each other. If the solution is allowed excess time in this state they would not be able to bond to the surface of the gold and effectively give no coating of the gold particles. Therefore, at this stage only thirty minutes is given between isolation of the oligonucleotides and mixture of the gold nanoparticle solution with DNA. For every batch of DNA-coated particles the following samples are created: GNPs with hairpin, GNPs with AS1411, and GNPs with both hairpin and AS1411. The sample with both hairpin and AS1411 molecules used an equal ratio of each oligonucleotide and each was added simultaneously to the gold solution. The calculations used to determine the volumes of each reactant to add to create said particles used the Beer-Lambert Law and the definition 
of molarity. Given the absorbance, extinction coefficient, and volumes of each reactant the number of molecules available can be determined. The extinction coefficients of the oligonucleotides is provided by the supplier and although the precise parameter for the GNPs has not been determined. Studies have shown spherical nanoparticles of silver and gold to have similar extinction coefficients on the order of $10^{9}$ to $10^{10} \mathrm{~L} / \mathrm{mol}-\mathrm{cm}$ (Navarro 2013). Due to the significantly lower volume of similarly sized plates the lower end of this estimation was used. Variation in this parameter ultimately only becomes a factor when looking at exact loading efficiencies, as any ratio of nanoplates to oligonucleotides will get some degree of coating. The limiting reactant can be determined and then used to calculate the volumes to add to create the three samples as mentioned. For this study a ratio of 1120 oligonucleotides per gold nanoparticle was used to ensure sufficient loading of DNA. It has been shown in previous studies, and is suggested here, that uncoated gold nanoplates are sensitive to aggregation from exposure to salt (Guo 2010). The oligonucleotide solutions are at a $10 \mathrm{mM} \mathrm{PB}$ concentration when added to the gold solution and is then diluted to $5 \mathrm{mM}$ with the addition of Nanopure water. Additionally, irreversible aggregation of GNPs onto the walls of containers was also mitigated by the use of low DNA-binding tubes, such as Lobind Eppendorf (Hamburg, Germany) centrifuge tubes. All further processes were done with all samples in parallel unless otherwise noted.

After combining the appropriate volumes of gold and oligonucleotides the samples are incubated at $4{ }^{\circ} \mathrm{C}$ and vortexed every thirty minutes for the first three to four hours and then further incubated at $4{ }^{\circ} \mathrm{C}$ overnight. On the next day the excess DNA is removed through serial centrifugations and washings. Centrifugation was performed using a Beckman Coulter (Brea, CA) Microfuge 16 set at 2500 xg for 4 minutes. The resulting 
colorless supernatant was collected and the pellet was resuspended in 5mM PB solution. The sample was then sonicated with a Misonix (Farmingdale, NY) Microson XL 2000 sonication probe 10 times in 1 second bursts and vortexed to fully resuspend any particles. This centrifuging, supernatant removal, and resuspension is performed three times for each sample. After this stage each sample and corresponding supernatant is analyzed by UVVis spectroscopy, DLS, and zeta potential measurement.

\section{F. Loading Doxorubicin Into Hairpin}

A $100 \mathrm{uM}$ solution of doxorubicin hydrochloride was created from dry power by dissolving $0.3 \mathrm{mg}$ into $5 \mathrm{~mL}$ of Nanopure water and a fraction of this stock was taken and diluted to $10 \mathrm{uM}$ for addition to GNP-DNA particles. Previous studies of doxorubicin loading at a concentration of $5 \mathrm{uM}$ for $300 \mathrm{nM}$ DNA hairpin showed saturation of the hairpin at 4 doxorubicin molecules per hairpin (Luo 2011). Due to the decreased stability of the gold nanoplates in high salt concentrations, to which doxorubicin hydrochloride contributes, the concentration of doxorubicin used to load the particles was limited to 0.5 uM. This will not provide saturation of the hairpin but will still allow for some loading of dox and provide a measurable fluorescence upon release. Maximizing dox loading is not crucial for proof of concept and preventing aggregation of the particles is crucial to further testing. To load the particles with doxorubicin a GNP-DNA sample is diluted to an absorbance, or optical density (OD), of 3 with a doxorubicin concentration of $0.5 \mathrm{uM}$ using $5 \mathrm{mM}$ PB as needed for dilution. For this step only GNPs with hairpin and GNPs with hairpin and AS1411 were used as GNPs with AS1411 have no ability to intercalate with dox, as it does not have repeating $\mathrm{C}-\mathrm{G}$ bases or traditional base pairing to provide the pi stacking arrangements. These samples were allowed 2 hours to react at room temperature, 
approximately $26^{\circ} \mathrm{C}$. To remove excess dox the samples were centrifuged and washed in a manner analogous to the DNA loading protocols. Centrifugation was performed at 1000 $\mathrm{xg}$ for 2 minutes or 4 minutes if no aggregation was seen. The largely colorless supernatant was collected leaving a purple pellet that is then resuspended with $5 \mathrm{mM}$ PB and vortexed. This process is performed up to 3 times if no aggregation occurs but was done twice in some cases to prevent significant loss of particles due to irreversible aggregation. Aggregation in previous samples was marked by loss of SPR bands, size analysis showing several fold increase, and zeta potential readings approaching zero. Visibly these changes were coincident with rapid change in color, from purple to blue or gray, and the collection of gray film or particulates on tube walls. Supernatants are analyzed for fluorescence to determine the amount of dox present, which can be used to estimate the number of hairpin particles present on the GNPs. The resulting particles are now GNPs functionalized with hairpin or hairpin/AS1411 oligonucleotides intercalated with doxorubicin. These samples are then analyzed for UV-Vis spectra for particle content and absorbance at $817 \mathrm{~nm}$.

\section{E. Laser-induced Release of Doxorubicin}

Using the nIR absorbance peak obtained from the UV-Vis spectrum the samples are diluted to $0.5 \mathrm{OD}$ and a volume of $0.5 \mathrm{~mL}$ using Nanopure water. Before laser application each sample is then analyzed for fluorescence along with a control, which has not been loaded with dox, similarly diluted from the same batch of functionalized particles. A fluorescence measurement was taken 3 times for each sample. In turn each sample was dispensed into a 48 well plate and heated with a laser. The $817 \mathrm{~nm}$ wavelength laser was set to $8200 \mathrm{~mA}$ $(1000 \mathrm{~mW})$ and emitted $21 \mathrm{~cm}$ above the target. The laser area consisted of a circle with a diameter of $1.25 \mathrm{~cm}$ covering the well fully. The actual laser power at the well plate was 
measured with a Coherent Laser Power Meter as approximately $800 \mathrm{~mW}$. Duration of laser application was 5 minutes followed by immediate collection and centrifugation at $1000 \mathrm{xg}$ for 2 to 4 minutes, matching the washing setting used to wash the sample in the dox loading process. The supernatant was collected and the pellet resuspended to $0.5 \mathrm{~mL}$ with Nanopure water and vortexed briefly. This process is performed 3 times to obtain a release profile of doxorubicin. Temperature is also measured during laser application using an Omega thermocouple probe and USB data logging device. After the final centrifugation and supernatant collection the 3 collected supernatants are centrifuged at $1000 \mathrm{xg}$ for 4 minutes to pellet any coincident gold in solution. Then $200 \mathrm{uL}$ from the top of each supernatant is analyzed for fluorescence, taking 3 measurements per sample. This procedure is done for 3 replicates of dox loaded and control samples of GNPs with hairpin and GNPs with hairpin and AS1411. 


\section{RESULTS AND DISCUSSION}

\section{A. Gold Nanoparticle Synthesis}

GNPs were produced using the Diasynth method and expected to contain a large fraction of plates, comparatively few spheres, and with a controllable SPR band within the NIR range. The spectrum of a sample used for subsequent functionalization, dox loading, and lasering are presented in FIGURE 6.The UV-Vis absorbance spectra show favorable SPR bands that will absorb highly within the therapeutic window and at the $817 \mathrm{~nm}$ wavelength of the available laser. The ratio of NIR band to $530 \mathrm{~nm}$ band can also approximate the ratio of plates to spheres. As nanospheres only exhibit SPR bands in the visible region and nanoplates exhibit in the nIR region these two can be easily differentiated within a spectra measurement. Typically the nIR peak should be 2 times as large as a $530 \mathrm{~nm}$ peak for a composition consisting of mainly plates (Patel 2012). The size for the nanoplates, identified as contributing to the majority of intensity readings from DLS, was found to be $68.46 \mathrm{~nm}$ on average, with a standard deviation of $2.74 \mathrm{~nm}$. The measured zeta potentials averaged $-31.48 \mathrm{mV}$ with a standard deviation of $0.95 \mathrm{mV}$. 


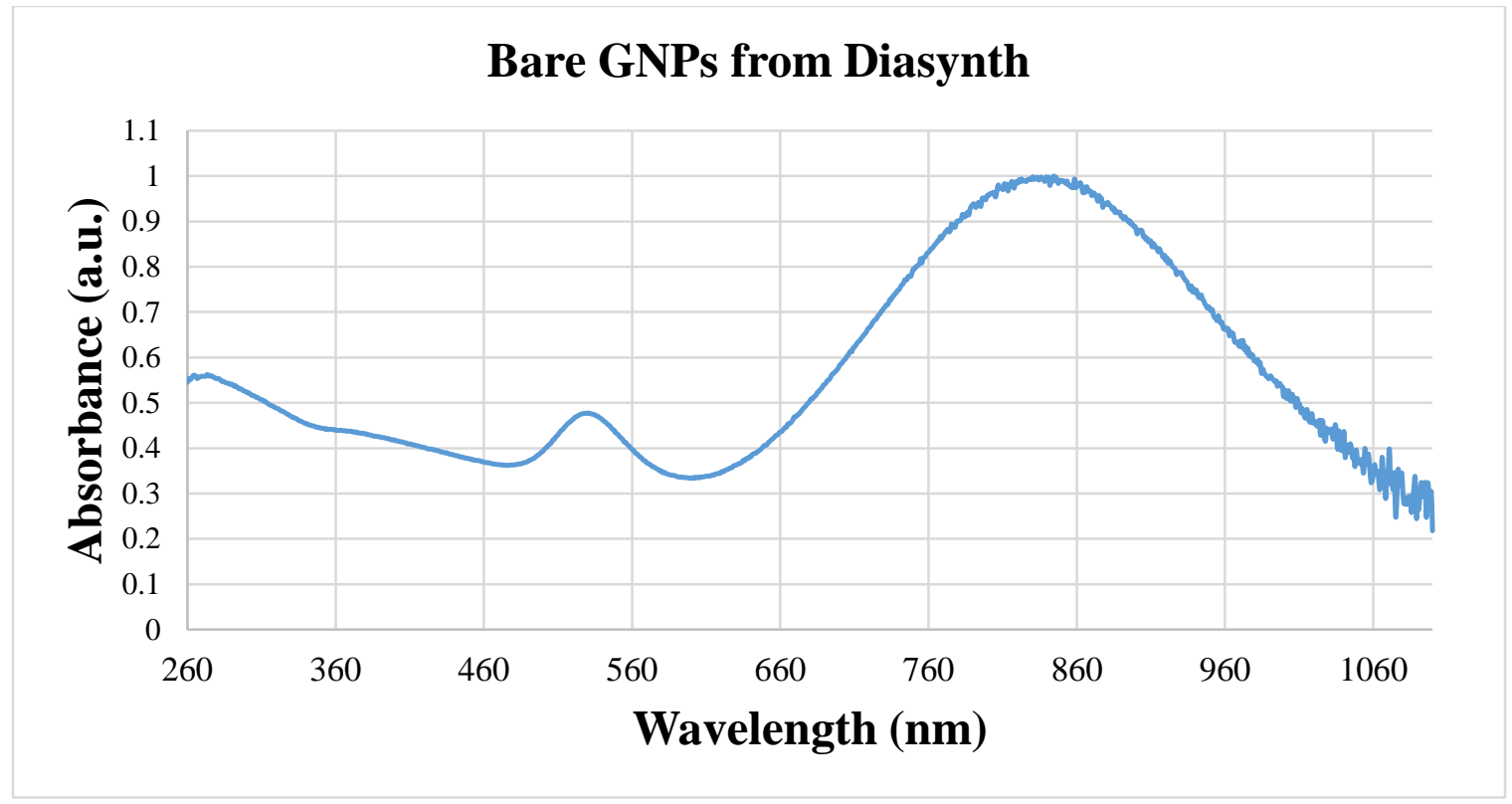

FIGURE 6 UV-Vis spectrum of representative gold nanoparticles synthesized using the Diasynth method. The two peaks approximate the ratio of spheres and plates. Absorbance in therapeutic windows indicates suitability for laser absorption.

Synthesis of GNPs using the Diasynth methods as described previously produced particle with SPR bands controllable through variation in temperature and dialysis membrane length. Other variations in the reactants such as supplier and duration in solution seem to cause changes in this response giving a variation in absolute characteristics but maintaining relatively predictable synthesis outcomes. Through iterative refinement of the optimal temperature and membrane length the SPR peak was tuned to obtain the particles detailed above. For the purposes of this research the spherical particles act as a contaminant, and theoretically should be functionalized in subsequent steps, while the practical focus is on the nanoplates. With further research into the characteristics, interactions, and application of gold nanoplates the isolation and processing of these particle can be further refined, but is outside of the scope of this project. The use of a fixed 
wavelength laser at the wavelength of max absorption for the plates is assumed to effectively only significantly heat the nanoplate population.

\section{B. Functionalization of GNPs with DNA}

The success of coating the GNPs with DNA was to be confirmed through change in the size and zeta potential of the particles. As shown in TABLE 2 the size increases across all samples after reacting with the oligonucleotides. The average size increase is 23.5, 42.23, and $26.13 \mathrm{~nm}$ for GNP-Hairpin, GNP-AS1411, and GNP-Hairpin-AS1411 respectively, with GNP-AS1411 having the highest standard deviation at $23.9 \mathrm{~nm}$. Zeta potential had more consistent data across samples with the average change in potential of $-11.90,-11.40$, and $-9.52 \mathrm{mV}$ respectively, with the highest standard deviation being 2.47 $\mathrm{mV}$. Analysis of the variance (Appendix Ia.) showed that there was a significant difference in size between bare and coated particles at a 95\% confidence level, while coating mixture was not a significant factor. Similarly, it shows a significant difference at a 95\% confidence

level in zeta potential for bare and coated particles but found the type of coating not a significant factor. 
TABLE 2 SIZE AND ZETA POTENTIAL OF DNA-FUNCTIONALIZED GNPS

\begin{tabular}{|c|c|c|c|c|c|c|}
\hline & \multicolumn{3}{|c|}{ Particle Size (nm) } & \multicolumn{3}{|c|}{ Zeta Potential (mV) } \\
\hline & Bare & $\begin{array}{l}\text { DNA- } \\
\text { coated }\end{array}$ & Change & Bare & $\begin{array}{l}\text { DNA- } \\
\text { coated } \\
\end{array}$ & Change \\
\hline GNP-Hairpin-1 & 70.40 & 95.66 & 25.26 & -30.8 & -44.15 & -13.35 \\
\hline GNP-Hairpin-2 & 70.40 & 100.55 & 30.15 & -30.80 & -44.10 & -13.30 \\
\hline GNP-Hairpin-3 & 66.52 & 81.62 & 15.1 & -32.15 & -41.20 & -9.05 \\
\hline $\begin{array}{l}\text { GNP-Hairpin Average } \\
\text { (Standard Deviation) }\end{array}$ & $\begin{array}{l}69.11 \\
(2.23) \\
\end{array}$ & $\begin{array}{l}92.61 \\
(9.83) \\
\end{array}$ & $\begin{array}{l}23.50 \\
(7.68) \\
\end{array}$ & $\begin{array}{l}-31.25 \\
(0.78) \\
\end{array}$ & $\begin{array}{r}-43.15 \\
(1.69) \\
\end{array}$ & $\begin{array}{c}-11.90 \\
(2.47) \\
\end{array}$ \\
\hline GNP-AS1411-1 & 70.40 & 85.63 & 15.23 & -30.80 & -40.25 & -9.45 \\
\hline GNP-AS1411-2 & 66.52 & 127.3 & 60.78 & -32.15 & --46.40 & -14.25 \\
\hline GNP-AS1411-3 & 66.52 & 117.2 & 50.68 & -32.15 & -42.65 & -10.5 \\
\hline $\begin{array}{l}\text { GNP-AS1411 Average } \\
\text { (Standard Deviation) }\end{array}$ & $\begin{array}{l}67.81 \\
(2.24) \\
\end{array}$ & $\begin{array}{l}110.04 \\
(21.74) \\
\end{array}$ & $\begin{array}{r}42.23 \\
(23.92) \\
\end{array}$ & $\begin{array}{l}-31.70 \\
(0.78) \\
\end{array}$ & $\begin{array}{r}-43.10 \\
(3.10) \\
\end{array}$ & $\begin{array}{c}-11.40 \\
(2.52) \\
\end{array}$ \\
\hline GNP-Hairpin-AS1411-1 & 70.40 & 94.7 & 24.3 & -30.8 & -40.70 & -9.90 \\
\hline GNP-Hairpin-AS1411-2 & 66.52 & 99.22 & 32.70 & -32.15 & -41.95 & -9.80 \\
\hline GNP-Hairpin-AS1411-3 & 66.52 & 87.92 & 21.40 & -32.15 & -41.00 & -8.85 \\
\hline $\begin{array}{c}\text { GNP-Hairpin-AS1411 } \\
\text { Average } \\
\text { (Standard Deviation) }\end{array}$ & $\begin{array}{l}67.81 \\
(2.24)\end{array}$ & $\begin{array}{l}93.95 \\
(5.69)\end{array}$ & $\begin{array}{l}26.13 \\
(5.87)\end{array}$ & $\begin{array}{l}-31.70 \\
(0.78)\end{array}$ & $\begin{array}{r}-41.22 \\
(0.65)\end{array}$ & $\begin{array}{r}-9.52 \\
(0.58)\end{array}$ \\
\hline
\end{tabular}

The overall trend that points to successful binding is the significant but small increase in size. This is as one would expect as the physical size of the complex is increasing with the addition of oligonucleotides onto all sides of the particle. Assuming a length of $0.33 \mathrm{~nm}$ per base (Mandelkern, 1981) the single stranded hairpin and AS1411 
would be $16.83 \mathrm{~nm}$ and $10.56 \mathrm{~nm}$ respectively. After folding into their respective structures of double strand and quadruplex they would then be approximately $8.42 \mathrm{~nm}$ and $2.64 \mathrm{~nm}$. Binding of these structures onto each side of the nanoparticle would then increase the overall size by at least $16 \mathrm{~nm}$ and $5 \mathrm{~nm}$ respectively. The possibility of crosslinking multiple oligonucleotides through base pairing or other interactions is an area of further research, but could create larger size increases. Additionally, the DLS reading perceives the hydration sphere of the particle taking into effect the interaction with water, thus increasing the apparent size of charged particles. Alternatively, any irreversible aggregation would be expected to increase the size much more dramatically and bring the zeta potential toward zero.

The UV-Vis spectra as seen in FIGURE 7 shows a set of coated particles that maintain a high SPR peak in the nIR range. This is crucial to the success of the particles as a large shift away from the laser frequency or a loss in absorbance would reduce the efficacy of these particles to heat with $817 \mathrm{~nm}$ laser application. The spectra changes associated with coating of the particles seems to vary little between coating composition and depends more on the source bare GNPs . This could include affinity for aggregation and presence of spherical particles. As mentioned previously the resulting particles do have some variation despite controllability in the placement of the nIR peak. 


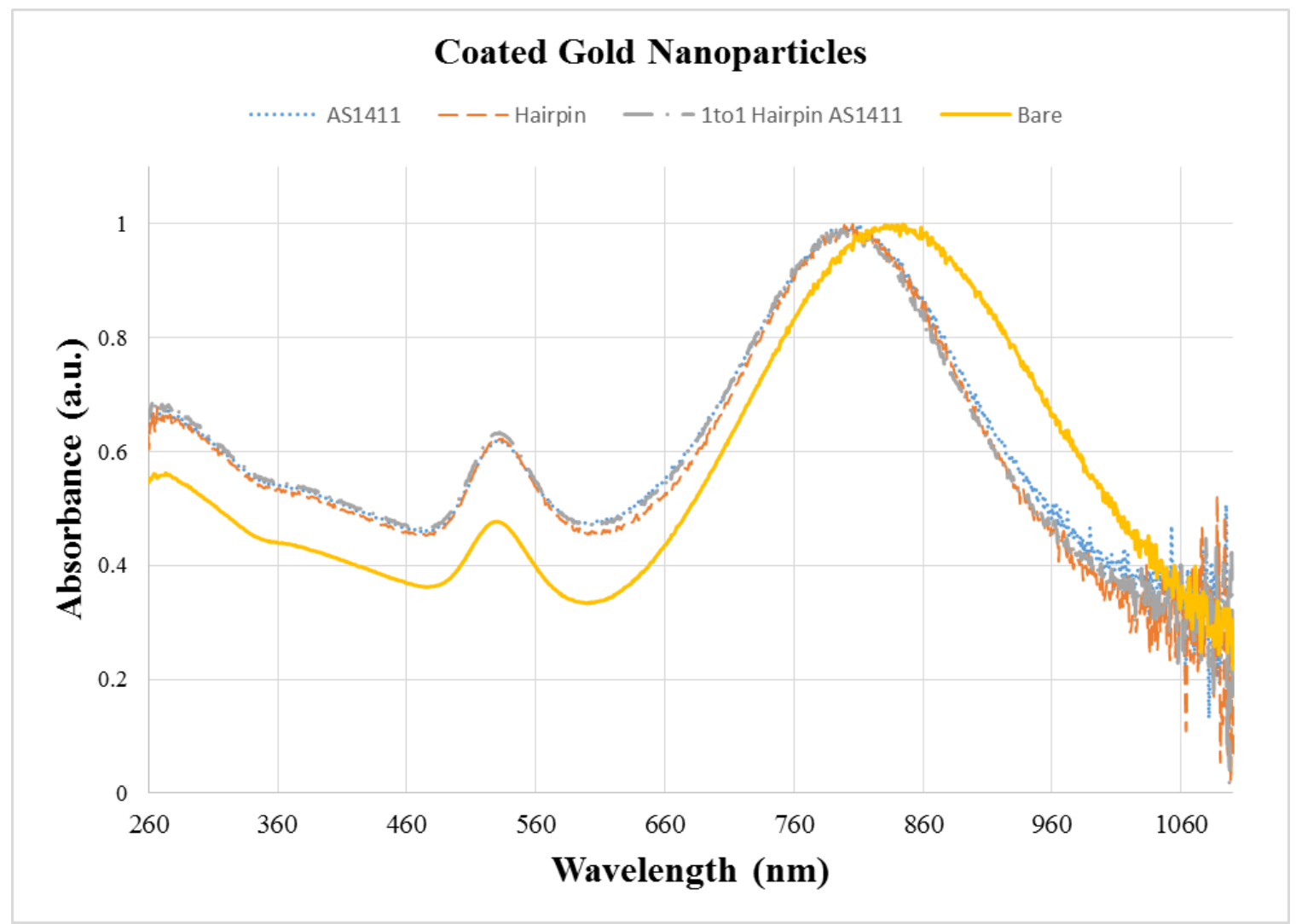

FIGURE 7 UV-Vis spectra of DNA-coated GNPs. The peaks shift slightly but maintain within the therapeutic window and absorbance at 817 is still suitable for laser absorption.

The procedure for functionalizing GNPs with oligonucleotides was taken from one study using similar approaches working with gold nanospheres (Luo 2011). The main difference between this study and those previously is that gold nanospheres have shown much more stability to the addition of salt where gold nanoplates tend to aggregate (Fan 2010). This is likely due to the geometry providing less uniform repulsion allowing for closer approach of plates along the planar faces leading to stacking. It has also been shown that the loading of DNA onto GNPs can be achieved through slow incremental increase in the salt concentration as high as $0.7 \mathrm{M} \mathrm{NaCl}$ for absolute maximum packing (Hurst 2006). 
However a portion of DNA will still bond to the particle surface without significant addition of salt. The occurrence of aggregation from salt addition or other incidental processes caused a visible change in the solution from a dark purple to blue or gray with eventual colorlessness and gray or black large particulates settling and adhering to the tube surface. This reaction can also be surmised from DLS measurements, showing a size change from less than $100 \mathrm{~nm}$ to several hundreds of nanometers as shown in TABLE 3, and UV-Vis spectra, shifting from a strong peak to a low broad absorbance with no discernable peak as seen in FIGURE 8. Due to the sensitivity of the nanoplates to high salt concentration, further processing was done with no additional salt added beyond the maintenance of a $5 \mathrm{mM}$ PB concentration. Possible methods of stabilization include capping agents, such as citrate, or coatings of long hydrophilic chains, such as polyethylene glycol. Such changes must be taken into consideration for the overall effect on the further processing and performance of the particles as these changes could have effects on the consistency, yield of production, and release of dox.

TABLE 3

SIZE AND ZETA POTENTIAL OF AGGREGATED DNA-FUNCTIONALIZED GNPS

\begin{tabular}{|c|c|c|}
\cline { 2 - 3 } \multicolumn{1}{c|}{} & \multicolumn{2}{c|}{ Particle Size $(\mathrm{nm})$} \\
\cline { 2 - 3 } \multicolumn{1}{c|}{} & Bare & DNA-coated \\
\hline GNP-Hairpin & 86.16 & 407.65 \\
\hline GNP-AS1411 & 86.16 & 354.75 \\
\hline GNP-Hairpin-AS1411 & 86.16 & 340.75 \\
\hline $\begin{array}{c}\text { GNP-DNA Average } \\
\text { (Standard Deviation) }\end{array}$ & $\mathbf{8 6 . 1 6}$ & $\begin{array}{c}\mathbf{3 6 7 . 7 2} \\
\mathbf{( 3 5 . 2 8})\end{array}$ \\
\hline
\end{tabular}




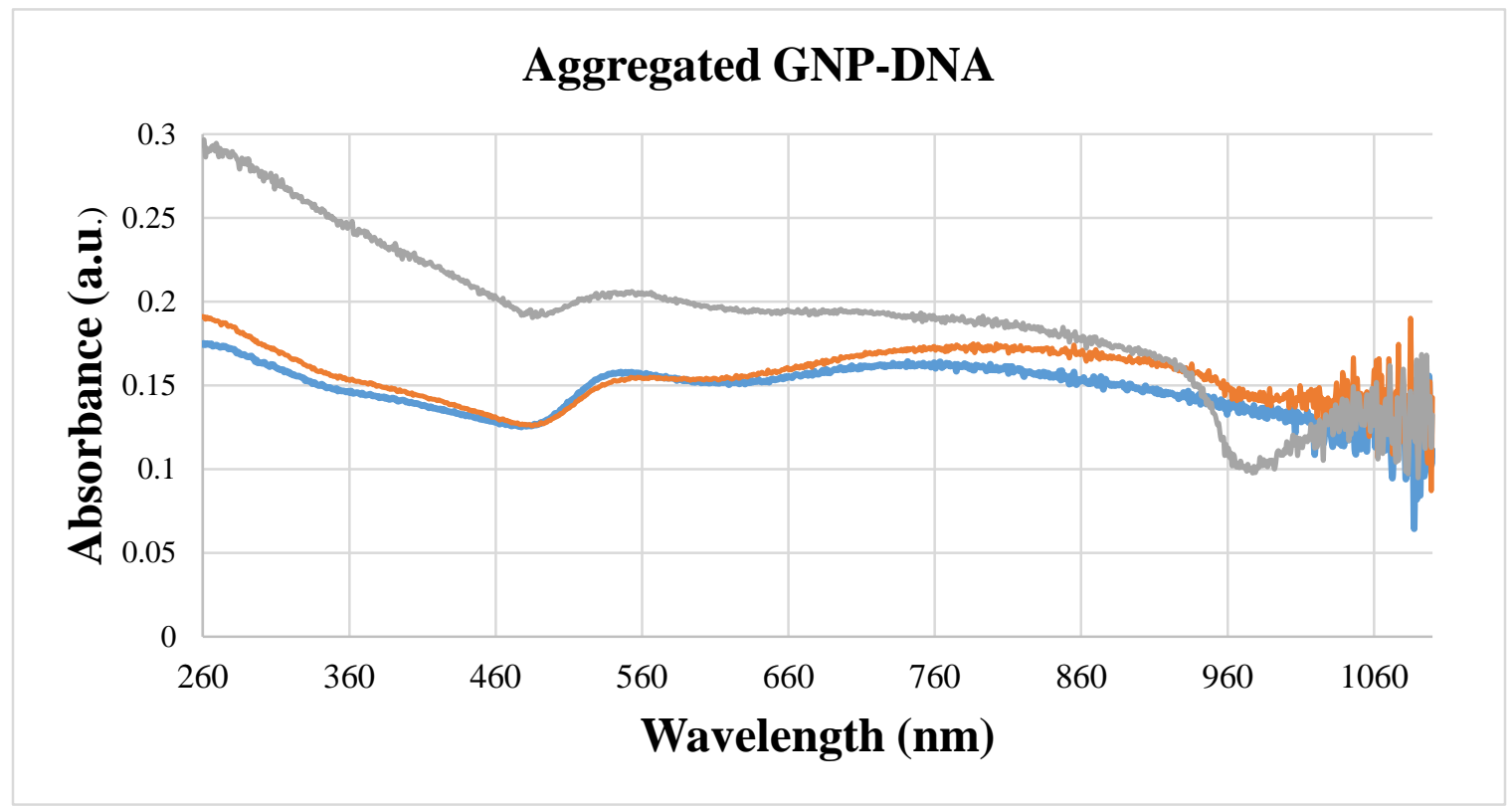

FIGURE 8 UV-Vis spectra of aggregated DNA functionalized GNPs. No substantial NIR peak is visible indicating the high absorbance plates are no longer present.

Despite attempts at quantification the amount of DNA loaded onto the GNPs could not be directly quantified at this time with the available reactants and analysis tools. Theoretically the $260 \mathrm{~nm}$ absorbance of supernatants from the washing of GNP-DNA particles would contain only absorbance from unbound DNA. However, this proved to be unrealistic as absorbance appeared higher than that of the DNA. This impossibly high reading indicated some other $260 \mathrm{~nm}$ absorbing particles, pointing to spherical gold remaining in the supernatant. Similarly, attempting to strip bound DNA from coated particles showed the same problem. DTT was used to break the thiol bond and release the DNA followed by centrifugation to pellet the GNPs while leaving the DNA in the supernatant. Again a second contributing factor appeared to be absorbing at $260 \mathrm{~nm}$ in addition to the DNA. However, because the previous study achieved saturation of this same hairpin, at 4 dox molecules per oligonucleotide, the number of hairpins can be estimated 
from the quantification of dox loading. This is easily determined through fluorescence and is covered more extensively in subsequent sections.

\section{B. Loading Doxorubicin into Hairpin}

After confirmation of DNA binding to the GNPs the hairpin molecules were loaded with dox for later release. As mentioned previously, attempts to use the concentration of dox found in other established protocols (Luo 2011) lead to aggregation of the particles due to high salt concentration. Therefore a reduced concentration of dox was used for loading. The analysis of the particles after loading and washing saw some decreases in overall quality of the nIR peak but not as severe as seen with aggregation. Additionally, during processing the visible color of the particles remained constant and minimal adherence to the tube walls was seen. FIGURE 9 shows a spectrum of a sample at this stage. Size and zeta measurements could not be reasonably taken at this stage due to volumetric constraints. 


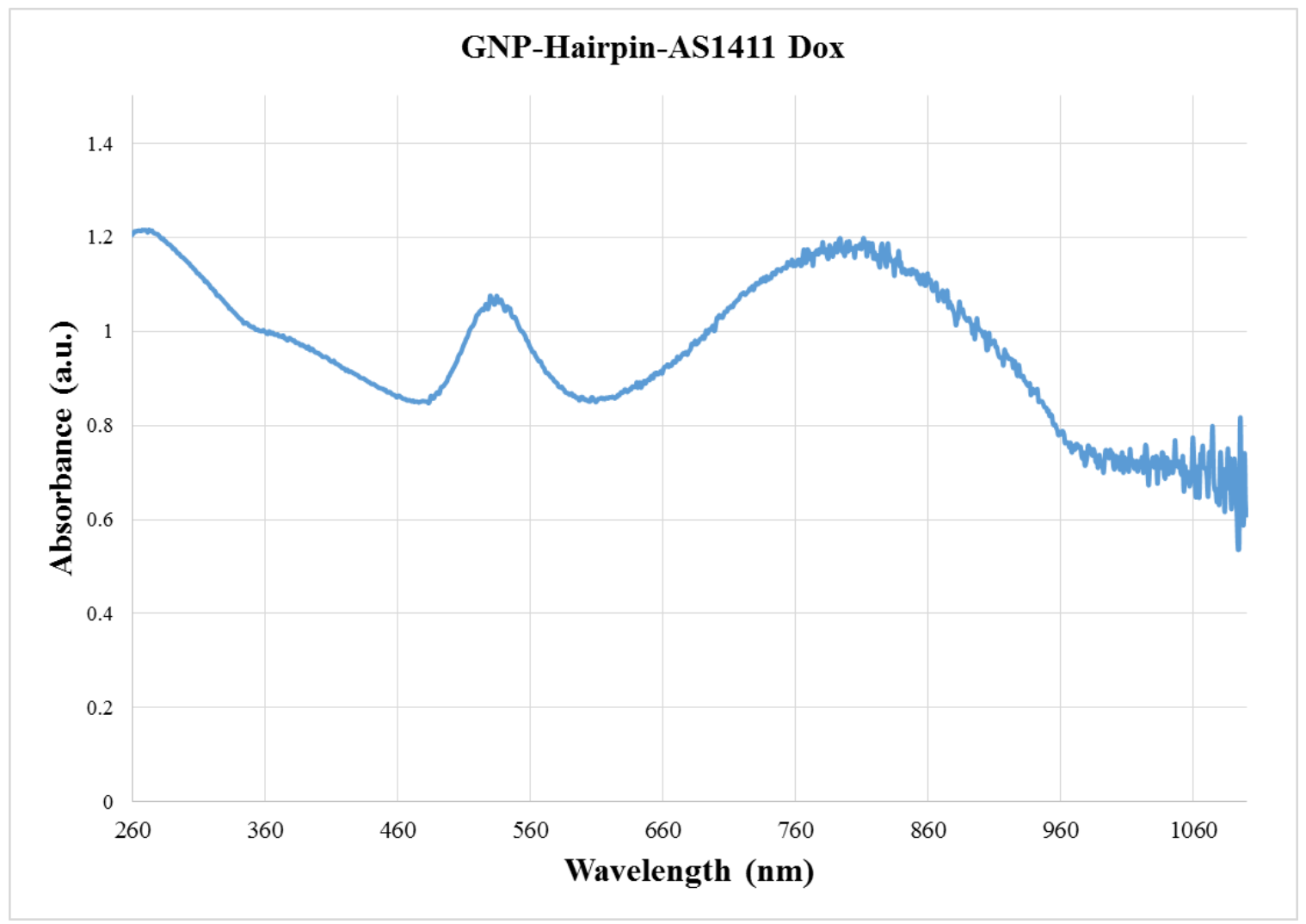

FIGURE 9 UV-Vis spectra of GNP-Hairpin-AS1411 loaded with dox. Aggregation effects disproportionately affect the plates causing a decrease in plate to sphere absorbance ratio

Dox loading was quantified indirectly by measuring the fluorescence of the wash supernatants. Using the raw fluorescence measurements and a fluorescence calibration curve (see Appendix 1D) for dox the concentration of dox in the supernatants can be determined. With an initial concentration of $0.5 \mathrm{uM}$ dox added during loading the dox concentration in the supernatant is assumed to be the difference between initial concentration and the loaded concentration. From this relationship the amount of successfully loaded dox can be calculated followed by total molecules of dox given the volume of supernatant. Then the known absorbance of loaded particles at $820 \mathrm{~nm}$ can be used again with the Beer-Lambert equation to determine the total number of nanoplates 
present in the volume of loaded particles. The number of dox molecule and gold nanoplates in the final sample have been found and can be used to find the number of dox per nanoplate. Conservatively assuming 1 molecule of dox per hairpin molecule, as Luo et al. found previously a saturation of 4 , the number of hairpins per gold nanoparticle can be calculated. The number of oligonucleotides per plate estimated from dox loading is detailed in TABLE 4.

TABLE 4

NUMBER OF HAIRPIN OLIGONUCLEOTIDES PER GOLD NANOPLATE

\begin{tabular}{|c|c|c|}
\hline Sample & GNP-Hairpin & GNP-Hairpin-AS1411 \\
\hline Replicate 1 & 369.6 & 370.5 \\
\hline Replicate 2 & 383.5 & 385.5 \\
\hline Replicate 3 & 385.4 & 386.3 \\
\hline Average Oligonucleotides per nanoplate & $\mathbf{3 7 9 . 5}$ & $\mathbf{3 8 0 . 8}$ \\
(Standard Deviation) & $\mathbf{( 8 . 6 )}$ & $\mathbf{( 8 . 9 )}$ \\
\hline
\end{tabular}

These results indicate a reasonable number of oligonucleotides, considering the indirect nature of the calculations. Because the hairpin-AS1411 sample is reacted with half the quantity of hairpin as the hairpin-only sample one would expect a noticeable decrease in dox loading capabilities but this is not seen. The difference between samples is relatively small potentially meaning that hairpin binding is preferential to AS1411 binding. Regardless of this difference there is evidence that there is loading of dox onto the hairpins which is crucial to the later release study.

This indirect type of quantification is not ideal but can serve at least as a check for sufficient dox washing. Seeing values indicative of high dox concentrations in supernatants would mean that free dox could still be in high concentration around the loaded particles. Assumptions inherent in this method of determining DNA loading include the previously 
mentioned extinction coefficient of gold nanoplates, relatively little interference of fluorescence by any gold nanospheres, and sufficient washing in previous steps. The theoretical physical limit of DNA loading can be approximated using surface area for a plate with edge length of $70 \mathrm{~nm}$, thickness of $2 \mathrm{~nm}$, and the diameter of a double helix of $2 \mathrm{~nm}$ (Mandelkern, 1981). This could give a plate total surface area of $7000 \mathrm{~nm}^{2}$, and at 90\% efficient hexagonal packing a maximum of 500 oligonulceotides per gold nanoplate. To better quantify the DNA loading other studies have used fluorescently labeled oligonucleotides and a similar approach could be used for further study of the loading characteristics of these particles.

\section{Laser-induced Release of Doxorubicin}

After loading the particles with dox the samples were exposed to laser light, at 817 $\mathrm{nm}$ wavelength, a power of $800 \mathrm{~mW}$, and duration of 5 minutes. The particles were then removed via centrifugation, and the amount of free dox in the supernatants quantified through fluorescence measurements. The samples showed a consistent release pattern despite some variations likely due to the salt sensitivity of the complexes. The average fluorescence reading after each lasering can be seen in FIGURE 10 for GNP-Hairpin and FIGURE 11 for GNP-Hairpin-AS1411. Also shown are the fluorescence readings from control GNP-DNA particles without dox that were lasered and processes in the same way. The percentage that each laser application contributed to the total release is also represented. The average fluorescence reading for the GNP-Hairpin particles is 336.6 , 227.4, and 167.6 FSU for the first, second, and third laser applications respectively. Meanwhile the control registered 140.1, 140.3, and 136.2 FSU for the same points. The GNP-Hairpin-AS1411 particles were a bit lower at 197.1, 155.4, and 142.9 FSU, with its 
control at 139.9, 129.2, and 128.1 FSU. The analysis of the variance of these results showed that significant factors at $95 \%$ confidence include laser number, loaded or non-loaded, and GNP-Hairpin or GNP-Hairpin-AS1411. This indicates that these all indeed effect the amount of dox released.

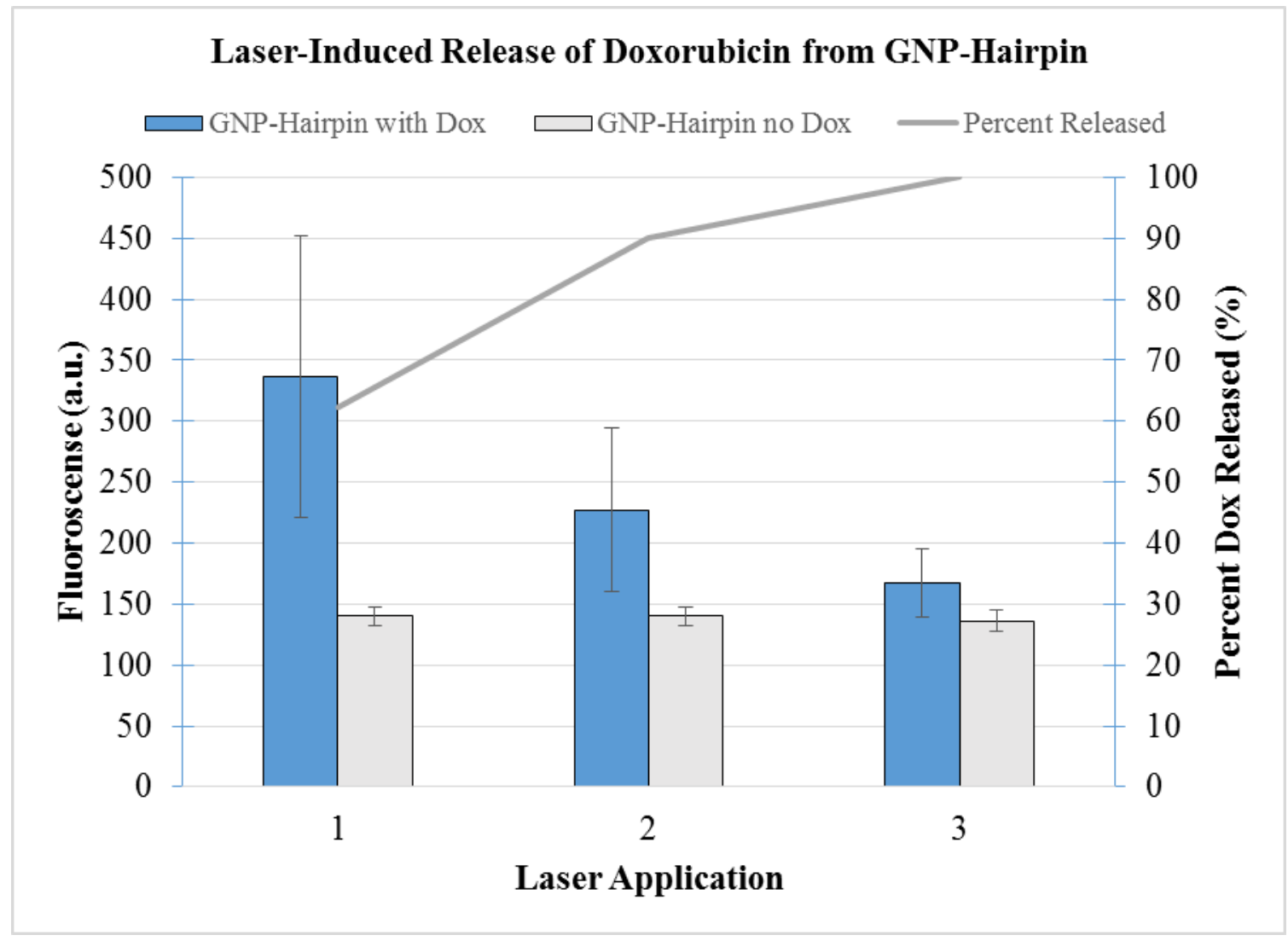

FIGURE 10 Fluorescence readings of supernatants from lasered GNP-Hairpin samples loaded with dox. The first laser releases the most dox and subsequent lasering approaches the control. Laser light exposure was $817 \mathrm{~nm}$ wavelength at $800 \mathrm{~mW}$ for 5 minutes. 


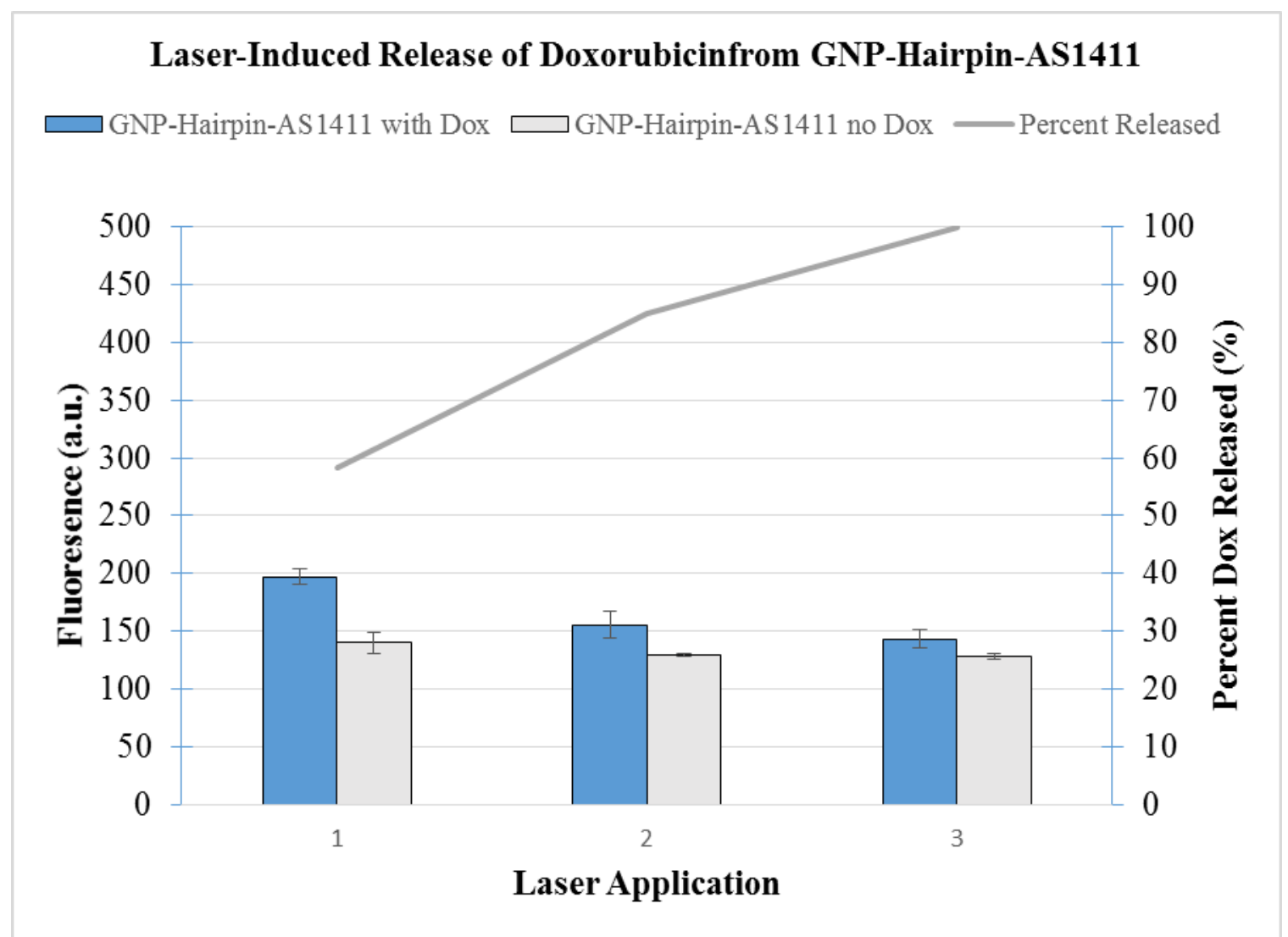

FIGURE 11 Fluorescence readings of supernatants from lasered GNP-Hairpin-AS1411 samples loaded with dox. The first laser releases the most dox and subsequent lasering approaches the control. Laser light exposure was $817 \mathrm{~nm}$ wavelength at $800 \mathrm{~mW}$ for 5 minutes

These results indicate that there was a release of dox due to the laser application and that the first laser application releases most of the dox. Analysis of the variance (Appendix I B) showed a significant difference between the dox loaded and control samples, and also indicated that the effects of laser number and coating type were significant to fluorescence measurement. This effectively serves as proof of concept that the particles do behave as proposed to serve as a possible vehicle for delivery and release of doxorubicin. One possible factor biasing these results could include the methodology of 
collecting the free dox after each lasering. The above results could be a symptom of dilution due to centrifugation and resuspension in the process thus showing fluorescence approaching the control. This is possible but should be mitigated by sufficient washing of excess dox during the loading phase. This specific possibility could be further studied by modifying the washing process, to more thoroughly ensure excess dox removal, and then release the dox with lasering to compare the results.

The other measurement taken during lasering was bulk solution temperature, seen in TABLE 5. All samples saw heating exceeding that of water with a maximum temperature of $58^{\circ} \mathrm{C}$ and minimum of $37.5^{\circ} \mathrm{C}$. The overall average was $46.3 \pm 7.6^{\circ} \mathrm{C}$ for particles loaded with dox and $43.4 \pm 3.7^{\circ} \mathrm{C}$ for particles with no dox. Analysis of the variance (Appendix I C) showed that there was no significant difference in the maximum temperature reached for any factor differentiating samples, including DNA coating, dox loading, or laser number. 
TABLE 5 Maximum temperatures during laser application

\begin{tabular}{|c|c|c|c|}
\cline { 2 - 4 } \multicolumn{1}{c|}{} & \multicolumn{3}{c|}{ Maximum Temperature $\left({ }^{\circ} \mathrm{C}\right)$} \\
\hline Sample & Laser 1 & Laser 2 & Laser 3 \\
\hline GNP-Hairpin-Dox 1 & 54 & 54.5 & 51 \\
\hline GNP-Hairpin-Dox 2 & 43.5 & 37.5 & 34 \\
\hline GNP-Hairpin-Dox 3 & 54.5 & 48 & 45 \\
\hline GNP-Hairpin-1 & 45 & 41 & 37.5 \\
\hline GNP-Hairpin-2 & 52 & 48 & 45.5 \\
\hline GNP-Hairpin-3 & 45 & 40.5 & 38 \\
\hline GNP-Hairpin-AS1411-Dox 1 & 40.5 & 40 & 38 \\
\hline GNP-Hairpin-AS1411-Dox 2 & 43 & 40.5 & 40.5 \\
\hline GNP-Hairpin-AS1411-Dox 3 & 58 & 57 & 53.5 \\
\hline GNP-Hairpin-AS1411-1 & 45 & 44 & 40.5 \\
\hline GNP-Hairpin-AS1411-2 & 47 & 43 & 39.5 \\
\hline GNP-Hairpin-AS1411-3 & 45 & 44 & 40.5 \\
\hline Water & 27 & 26 & 26 \\
\hline
\end{tabular}

These findings confirm that all samples were heated nearly the same while the variation could not be attributed to one particular factor. This likely means that any variation comes from processes in the production of the coated GNPs or even the production of the bare particles. Ultimately it is most important that the gold particles be heated enough to denature the hairpin structure to allow dox to be released. However the bulk temperature read here is not seen to be above $60^{\circ} \mathrm{C}$, well below the melting temperature $\left(\mathrm{T}_{\mathrm{M}}\right)$ of the hairpin at $69.7^{\circ} \mathrm{C}$. The important realization here is that the local heating at the particle surface induced by the SPR effect is effectively dampened by the large volume of water into which the heat has to dissipate, and therefore oligonucleotides conjugated to the particle surface likely experience greater thermal fluctuations compared to the bulk solutions. One study found the bulk temperature induced by this effect to be as high as $100{ }^{\circ} \mathrm{C}$ (Gobin 2010). This is also confirmed by the fact that despite bulk temperate 
of the solution being lower than the $\mathrm{T}_{\mathrm{M}}$ for the hairpin, significant dox release is still seen, which means that hairpin must be denaturing. The ability to effectively achieve hairpin denaturation and dox release at bulk temperatures near $43^{\circ} \mathrm{C}$ is very useful in a practical sense for achieving hyperthermic ablation of cancer cells without causing significant heatrelated damage to surrounding tissue (Kobayashi 2011). This data is also useful for future studies in correlating bulk temperature to the amount of dox released over time, with temperature being an easily measurable metric.

\section{FUTURE WORK}

The results presented here serve as a first look at the possibility of using gold nanoplates as a delivery system for multiple chemotherapeutic agents. Although the data presented here shows promise there is much left work to be done before any significant need can be met. Overall the data presented would benefit from more samples and refinement of the individual processes to further analyze the therapeutic potential of these particles. Other studies on the underlying processes of the related work, such as oligonucleotide packing on gold nanoplates or preferential binding of different oligonucleotides would also be greatly beneficial. Probably the most commonly found problem in this study was the aggregation of the gold nanoplates. This process has been studied with other GNPs but because not much has been done with pure nanoplates there is not much guidance for preventing aggregation. The methods and data presented here only represent a small subset of the potential ways of coating these particle, so it is very likely another method could cause less aggregation. A method of resisting aggregation in the presence of salt would allow for a much more controllable loading of oligonucleotides as mentioned previously. This would allow for a controllable maximum dox load to 
simplify future studies looking at oligonucleotide loading, dox loading, or even needed therapeutic concentrations of particles. Precisely determining the extinction coefficient of gold nanoplates would allow for a more accurate calculation for the number of gold nanoplates in a given solution.

The isolation of gold nanoplates or the synthesis of pure gold nanoplates is another thing that is currently being researched. As mentioned other techniques to analyze the loading of both DNA and dox would shed light on some of the possible hurdles encountered in this project. Ultimately, assuming continued interest and feasibility in this type of delivery vehicle, the next step after process optimization is live studies studying the efficacy of particles against cancer. This would include in vitro studies of cancerous and healthy cells, examining both cytotoxicity upon release to cancer and biocompatibility with healthy cells. Beyond that realm would be clinical studies in animals, and eventually humans, before being a new tool in the treatment of cancer. Even if that is not the final end point of this specific research it is sure to help the overall research into the possibilities of GNPs and nanoparticle drug delivery vehicles.

\section{CONCLUSION}

The work presented here has attempted to prove the potential of gold nanoplates coated with DNA oligonucleotides as a delivery vehicle for the controlled delivery of cancer drugs. GNPs with an SPR band in the nIR range were synthesized using the Diasynth method to preferentially separate nanoplates from nanospheres. The particles were then coated with two different DNA oligonucleotides with corresponding increases in particle size and more negative zeta potential. Doxorubicin was then intercalated into the attached DNA which was confirmed via fluorescence measurements. Laser induced 
release of doxorubicin from DNA was shown in bench top tests through fluorescence measurements.

Stability of gold nanoplates continues to be area in need of research as the optimal processing of these particles become ever more valuable. The processes presented here produced the proof of concept for these particles, but much room remains for optimization or modification. Further research into each aspect of the principles driving these reactions is needed to better quantify and understand these processes. Modification to enhance the abilities of these particles can also be explored as much work has already been done with gold nanospheres as drug delivery vehicles.

After refining the synthesis process in vitro cell studies are the next step for testing the therapeutic potential of these particles. The promise of a selectively released chemotherapy drug with multiple molecules to treat cancer is a great improvement over traditional chemotherapy, greatly reducing the side effects while potentially increasing the efficacy. The ultimate potential of this research is ultimately the full production and use of these or similar particles for cancer therapy in patients. 


\section{APPENDIX}

A. DNA Loading Statistical Analysis

\section{General Factorial Regression: Size versus Blocks, Oligos, Stage}

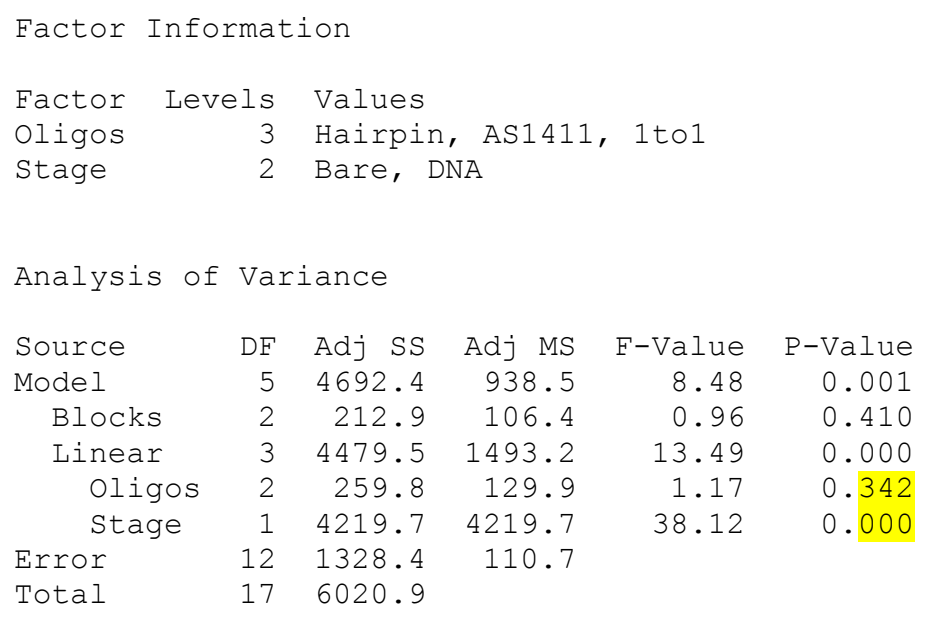

\section{Residual Plots for Size}
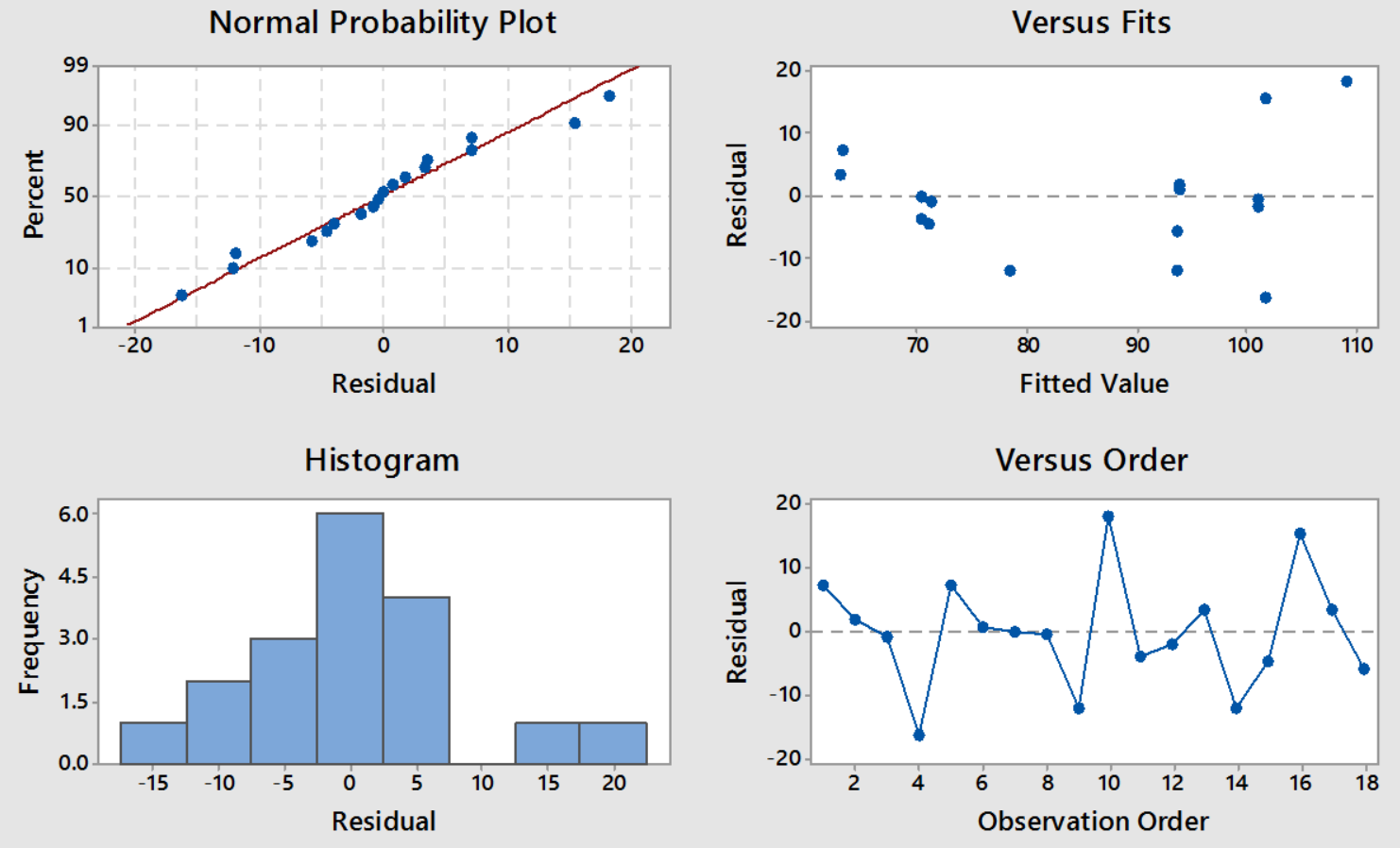

FIGURE 12 Residual plots for the size analysis of DNA loading

General Factorial Regression: Zeta versus Blocks, Oligos, Stage

Factor Information 


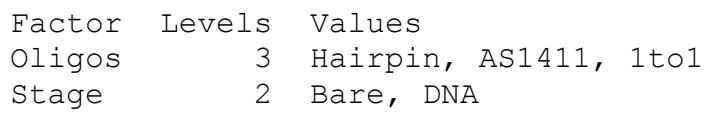

$\begin{array}{lrrrrr}\text { Source } & \text { DF } & \text { Adj SS } & \text { Adj MS } & \text { F-Value } & \text { P-Value } \\ \text { Model } & 5 & 550.004 & 110.001 & 51.62 & 0.000 \\ \quad \text { Blocks } & 2 & 8.584 & 4.292 & 2.01 & 0.176 \\ \quad \text { Linear } & 3 & 541.420 & 180.473 & 84.69 & 0.000 \\ \quad \text { Oligos } & 2 & 2.954 & 1.477 & 0.69 & 0.519 \\ \quad \text { Stage } & 1 & 538.467 & 538.467 & 252.69 & 0.000 \\ \text { Error } & 12 & 25.572 & 2.131 & & \\ \text { Total } & 17 & 575.576 & & & \end{array}$

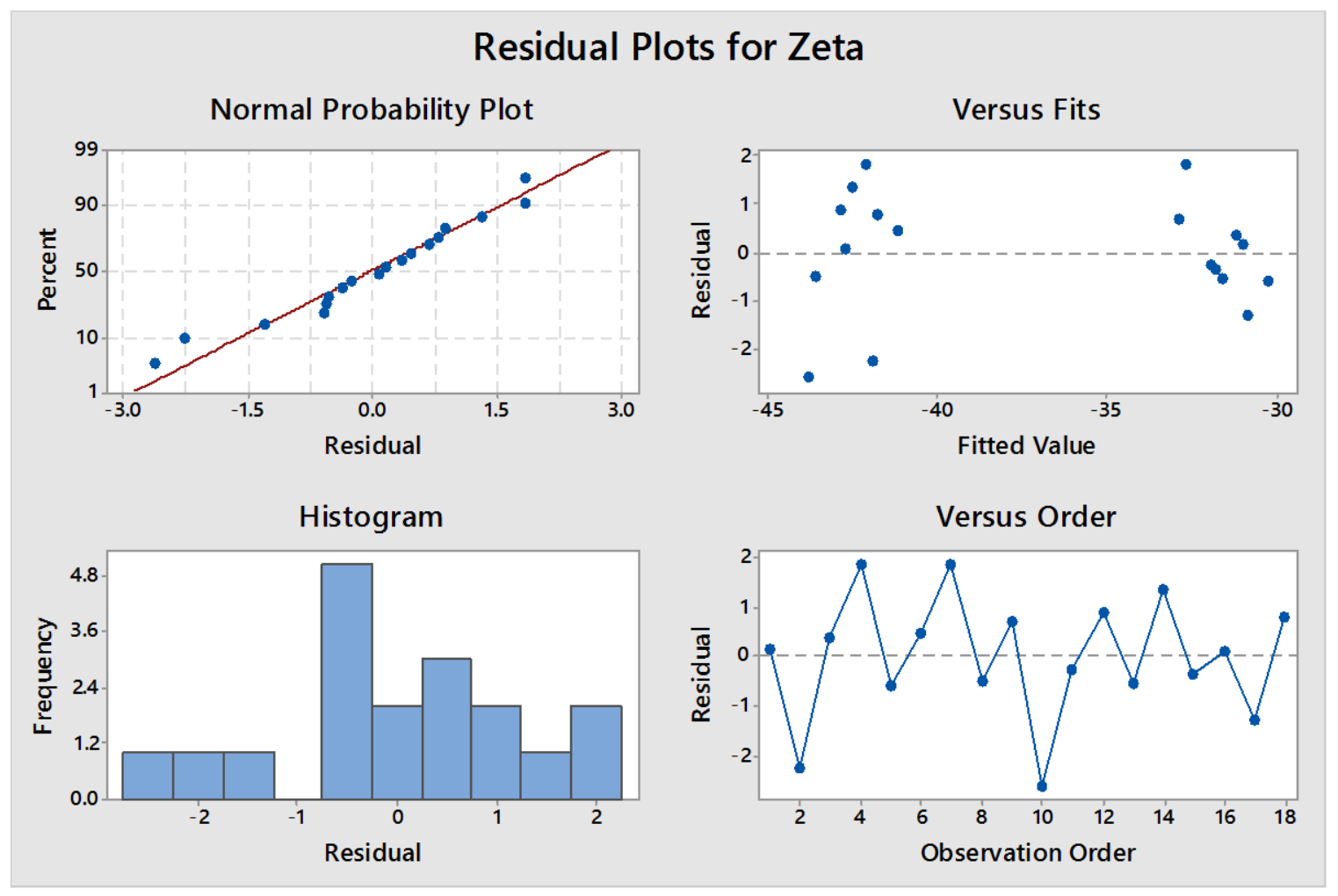

FIGURE 13 Residual plots for the zeta analysis of DNA loading 
B. Laser Induced Dox Release Statistical Analysis

\section{General Factorial Regression: FSU versus Dox, Hairpin or 1to1, Laser number}

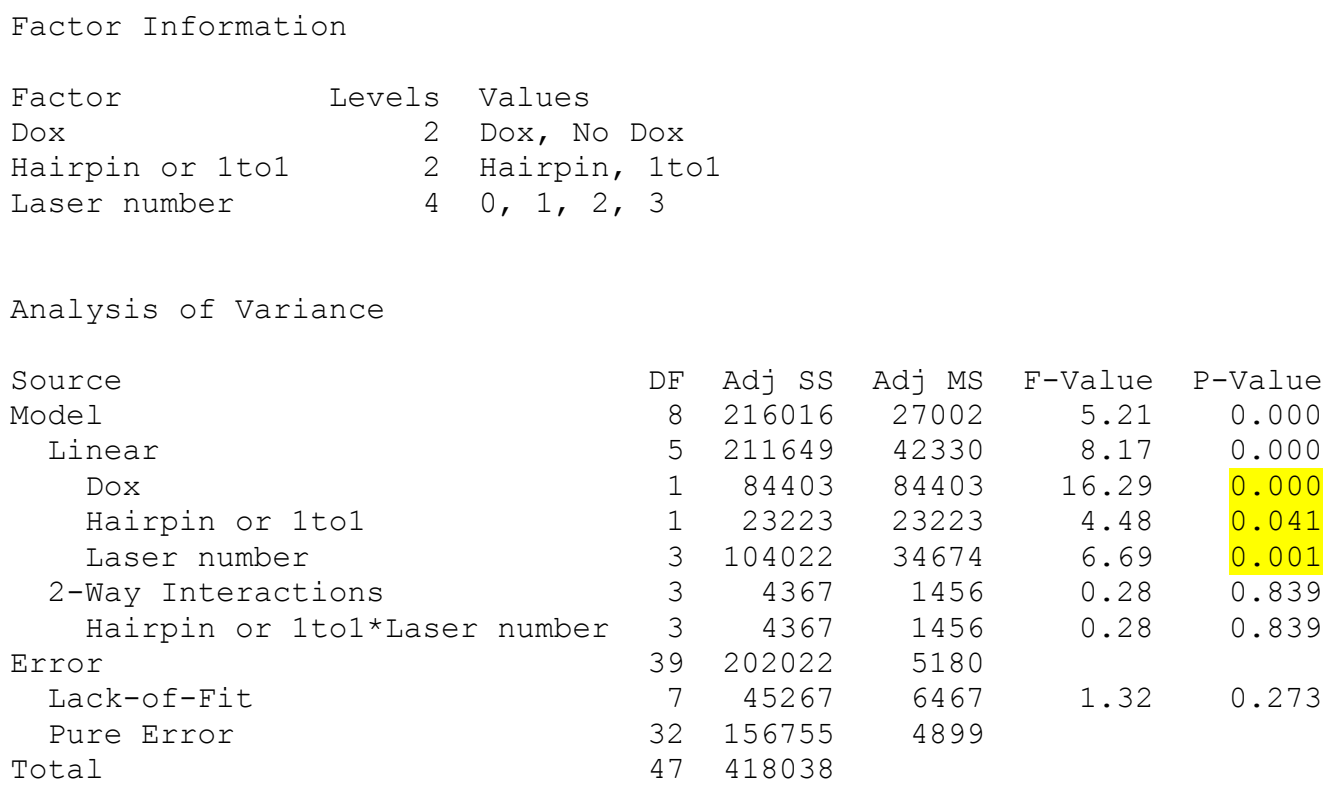

\section{Residual Plots for FSU}
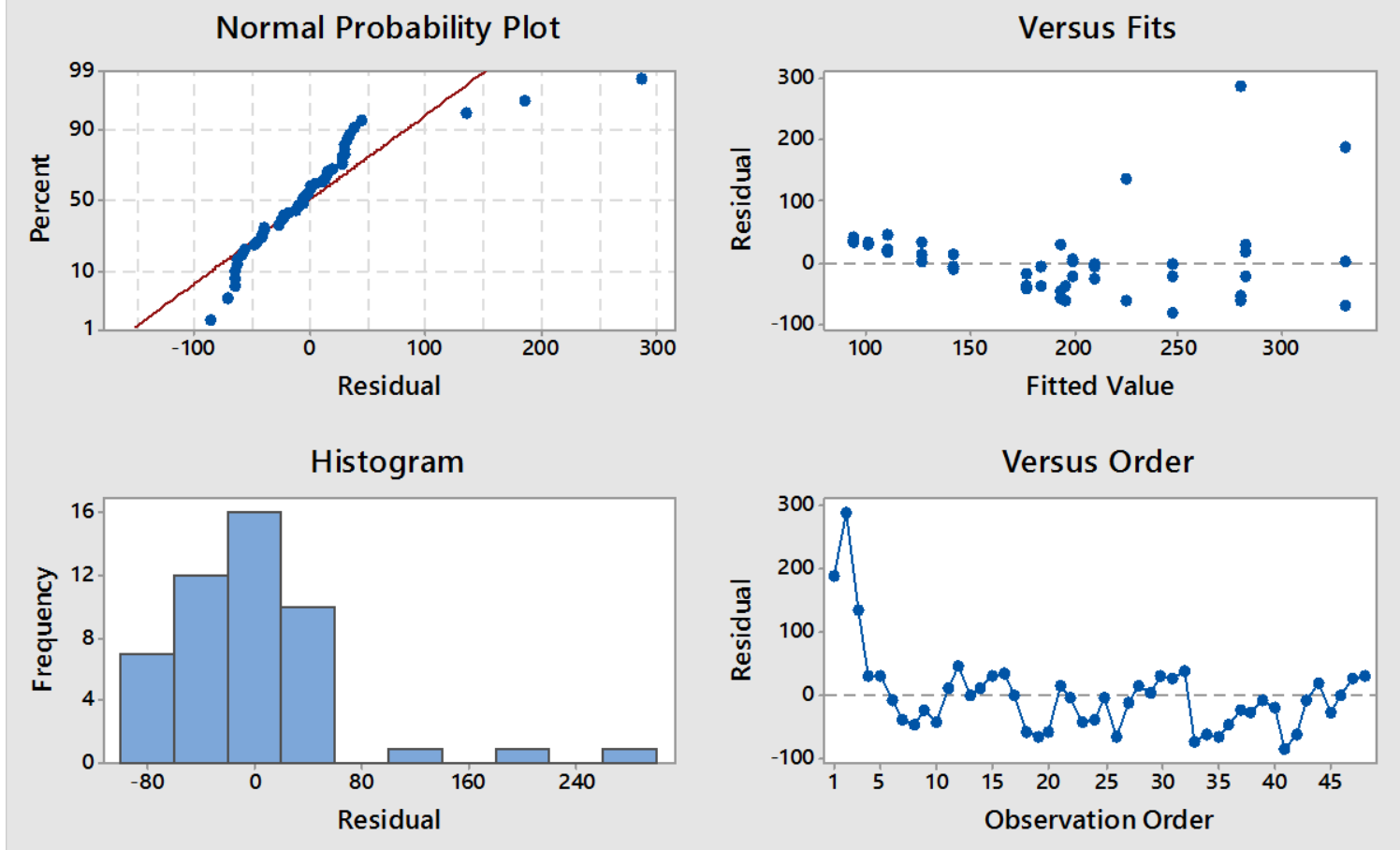

FIGURE 14 Residual plots for statistical analysis of dox release 


\section{Temperature Data Analysis}

\section{Multilevel Factorial Design}

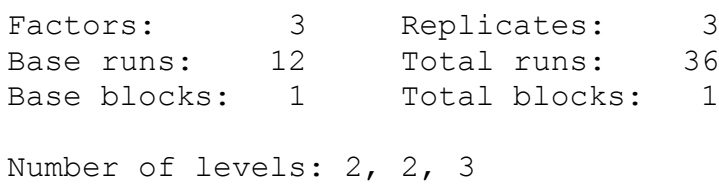

General Factorial Regression: Temp versus Dox, DNA, Laser

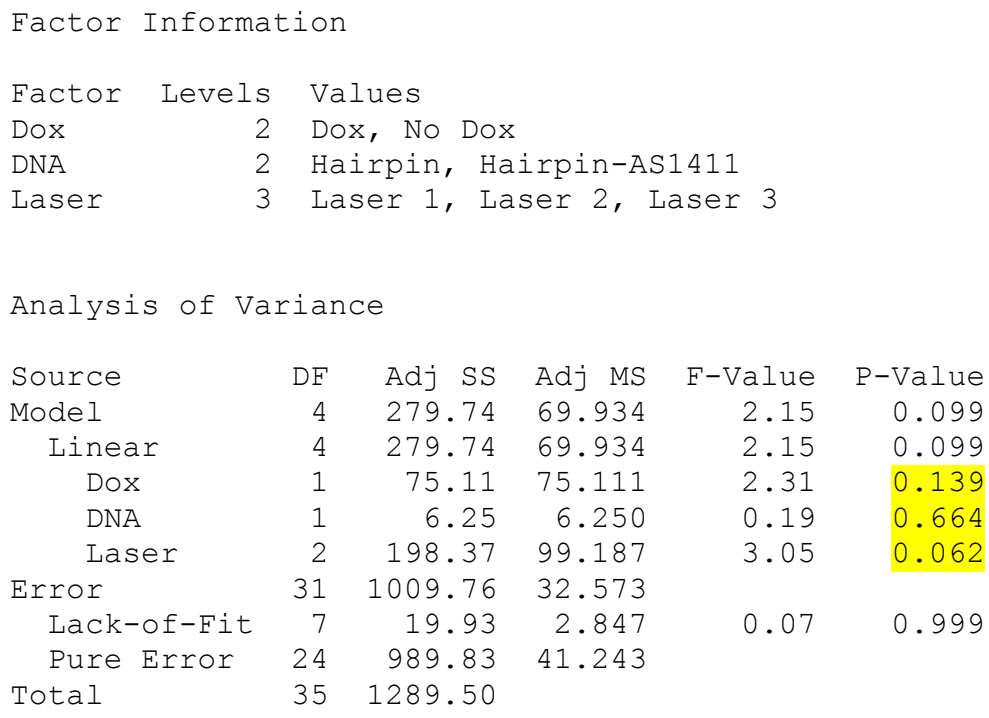




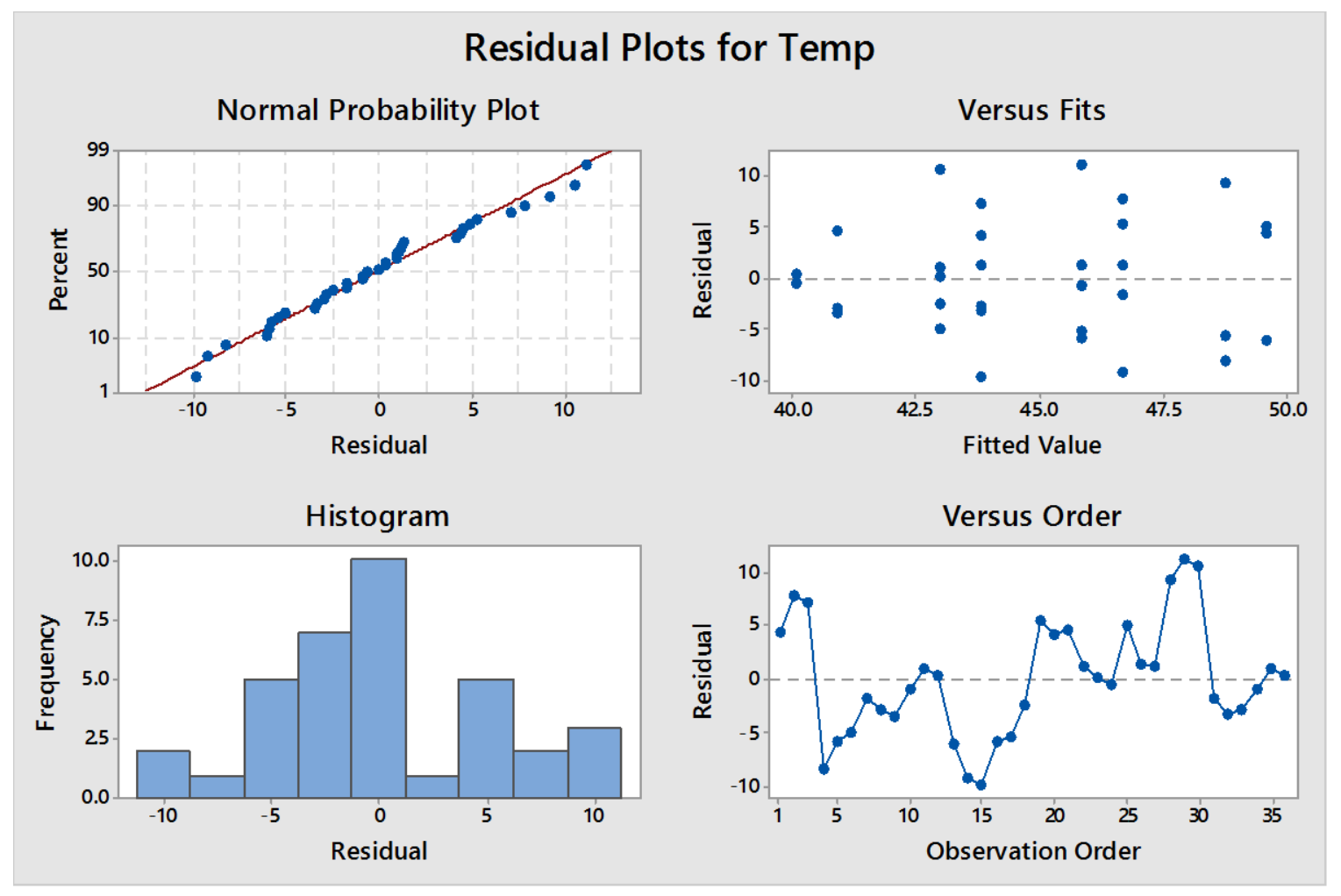

FIGURE 15 Residuals for temperature analysis

D. Doxorubicin calibration curve

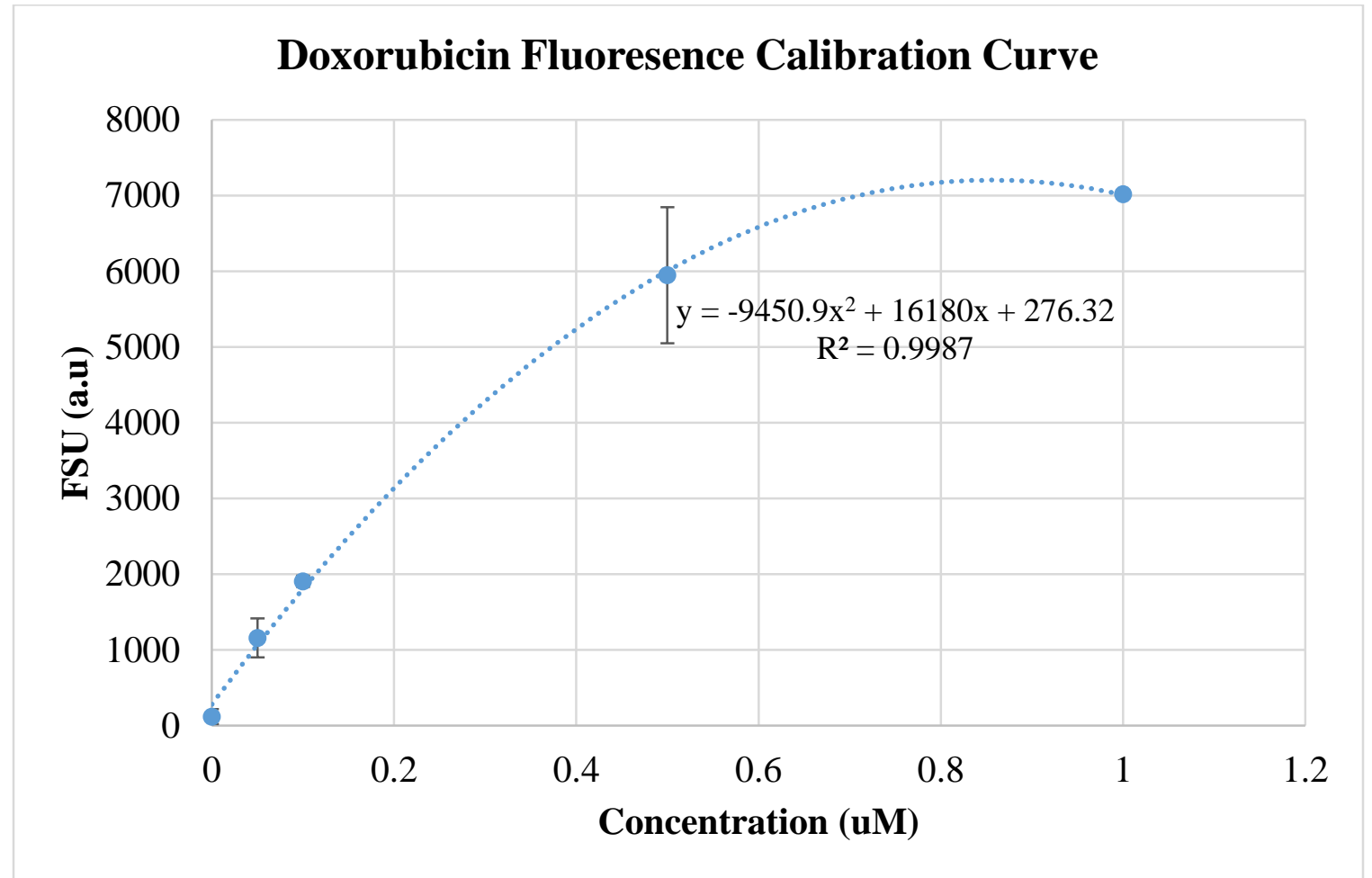

FIGURE 16 Fluorescence calibration curve for Doxorubicin 


\section{REFERENCES}

Agudelo, D., et al. (2014). "Intercalation of antitumor drug doxorubicin and its analogue by DNA duplex: structural features and biological implications." Int J Biol Macromol 66: 144-150.

Bates, P. J., et al. (2009). "Discovery and development of the G-rich oligonucleotide AS1411 as a novel treatment for cancer." Exp Mol Pathol 86(3): 151-164.

Bode, A. M. and Z. Dong (2009). "Cancer prevention research - then and now." Nat Rev Cancer 9(7): 508-516.

Boisselier, E. and D. Astruc (2009). "Gold nanoparticles in nanomedicine: preparations, imaging, diagnostics, therapies and toxicity." Chem Soc Rev 38(6): 1759-1782.

Broude, N. E. (2002). "Stem-loop oligonucleotides: a robust tool for molecular biology and biotechnology." Trends Biotechnol 20(6): 249-256.

Chabner, B. A. and T. G. Roberts, Jr. (2005). "Timeline: Chemotherapy and the war on cancer." Nat Rev Cancer 5(1): 65-72.

Delong, R. K., et al. (2010). "Functionalized gold nanoparticles for the binding, stabilization, and delivery of therapeutic DNA, RNA, and other biological macromolecules." Nanotechnol Sci Appl 3: 53-63.

Fan, X., et al. (2010). "Size-controlled growth of colloidal gold nanoplates and their highpurity acquisition." Nanotechnology 21(10): 105602.

Frederick, C. A., et al. (1990). "Structural comparison of anticancer drug-DNA complexes: adriamycin and daunomycin." Biochemistry 29(10): 2538-2549.

Ghosh, P., et al. (2008). "Gold nanoparticles in delivery applications." Adv Drug Deliv Rev 60(11): 1307-1315.

Gobin, A. M., et al. (2010). "Near-infrared-resonant gold/gold sulfide nanoparticles as a photothermal cancer therapeutic agent." Small 6(6): 745-752.

Guo, Z., et al. (2010). "Achieving high-purity colloidal gold nanoprisms and their application as biosensing platforms." J Colloid Interface Sci 348(1): 29-36.

Hirsch, L. R., et al. (2003). "Nanoshell-mediated near-infrared thermal therapy of tumors under magnetic resonance guidance." Proc Natl Acad Sci U S A 100(23): 13549-13554.

Hurst, S. J., et al. (2006). "Maximizing DNA loading on a range of gold nanoparticle sizes." Anal Chem 78(24): 8313-8318. 
Iyer, A. K., et al. (2006). "Exploiting the enhanced permeability and retention effect for tumor targeting." Drug Discov Today 11(17-18): 812-818.

James, K. (2013). Production Of High Yield Gold/Gold-Sulfide Nanoparticles Via Cellulose Membrane. Bioengineering, University of Louisville. Master of Engineering.

Kobayashi, T. (2011). "Cancer hyperthermia using magnetic nanoparticles." Biotechnol J 6(11): 1342-1347.

Luo, Y. L., et al. (2011). "Release of photoactivatable drugs from plasmonic nanoparticles for targeted cancer therapy." ACS Nano 5(10): 7796-7804.

Mandelkern, M., et al. (1981). "The dimensions of DNA in solution." J Mol Biol 152(1): 153-161.

Millstone, J. E., et al. (2009). "Colloidal gold and silver triangular nanoprisms." Small 5(6): 646-664.

Navarro, J. R. and M. H. Werts (2013). "Resonant light scattering spectroscopy of gold, silver and gold-silver alloy nanoparticles and optical detection in microfluidic channels." Analyst 138(2): 583-592.

Patel, D. (2012). A Novel High Yield Process For Gold Sulfide Nanoparticle Synthesis Via Shifting Equilibrium of Self-Assembling Reaction. Bioengineering, University of Louisville. Master of Engineering.

Song, J., et al. (2010). "Self-aggregation of oligonucleotide-functionalized gold nanoparticles and its applications for highly sensitive detection of DNA." Chem Commun $\underline{(\mathrm{Camb})}$ 46(30): 5548-5550.

Stahel, R. A. and U. Zangemeister-Wittke (2003). "Antisense oligonucleotides for cancer therapy-an overview." Lung Cancer 41 Suppl 1: S81-88.

Tacar, O., et al. (2013). "Doxorubicin: an update on anticancer molecular action, toxicity and novel drug delivery systems." J Pharm Pharmacol 65(2): 157-170.

Walter, N. G. and J. M. Burke (1998). "The hairpin ribozyme: structure, assembly and catalysis." Curr Opin Chem Biol 2(2): 303.

Weissleder, R. (2001). "A clearer vision for in vivo imaging." Nat Biotechnol 19(4): 316317.

Witten, K. G., et al. (2008). "Assembly of DNA-functionalized gold nanoparticles studied by UV/Vis-spectroscopy and dynamic light scattering." Phys Chem Chem Phys 10(14): 1870-1875. 
Zhang, G., et al. (2012). "Tunability and stability of gold nanoparticles obtained from chloroauric Acid and sodium thiosulfate reaction." Nanoscale Res Lett 7(1): 337. 